\title{
GLOBALLY F-REGULAR TYPE OF MODULI SPACES
}

\author{
XIAOTAO SUN AND MINGSHUO ZHOU
}

\begin{abstract}
We prove moduli spaces of semistable parabolic bundles and generalized parabolic sheaves with fixed determinant on a smooth projective curve are globally $F$-regular type.
\end{abstract}

\section{INTRODUCTION}

Let $X$ be a variety over a perfect field $k$ of characteristic $p>0$ and $F: X \rightarrow X$ be the Frobenius morphism. The $X$ is called $F$-split (Frobenius split) if the natural homomorphism $\mathcal{O}_{X} \hookrightarrow F_{*} \mathcal{O}_{X}$ is split. Although most of projective varieties are not $F$-split, some important varieties are $F$-split. For example, flag varieties and their Schubert subvarieties (cf. [7], [12]), the product of two flag varieties for the same group $G$ (cf. [8]) and cotangent bundles of flag varieties (cf. [4]) are proved to be $F$-split. An example, which is more closer to this article, should be mentioned. Mehta-Ramadas proved in [6] that for a generic smooth projective curve $C$ of genus $g$ over an algebraically closed field of characteristic $p \geq 5$, the moduli space of semistable parabolic bundles of rank 2 on $C$ is $F$-split, and made conjecture that moduli spaces of semistable parabolic bundles of rank 2 on any smooth curve $C$ with a fixed determinant is F-split.

The notion of globally $F$-regular variety was introduced by K. E. Smith in [15], a variety $X$ is called globally $F$-regular if for any effective divisor $D$, the natural homomorphism $\mathcal{O}_{X} \hookrightarrow F_{*}^{e} \mathcal{O}_{X}(D)$ is split for some integer $e>0$. It is clear that globally $F$-regular varieties must be $F$-split. Also, some well-known $\mathrm{F}$-split varieties include toric varieties and Schubert varieties are proved ([15], [5]) to be globally Fregular. Thus it is natural to extend Mehta-Ramadas conjecture: the moduli spaces $\mathcal{U}_{C, \omega}^{L}$ of semistable parabolic bundles of rank $r$ with a

Date: September 18, 2019.

1991 Mathematics Subject Classification. Algebraic Geometry, 14H60, 14D20.

Key words and phrases. Frobenius split, Moduli spaces, Parabolic sheaves.

Both authors are supported by the National Natural Science Foundation of China No.11831013; Mingshuo Zhou is also supported by the National Natural Science Foundation of China No.11501154. 
fixed determinant $L$ on any smooth curves $C$ (parabolic structures determined by a given data ) are globally F-regular varieties. It remains to be a very difficult open problem, we will study its characteristic zero analogy in this article.

A variety $X$ over a field of characteristic zero is called globally $F$ regular type (resp. $F$-split type) if its modulo $p$ reduction $X_{p}$ is globally $F$-regular (resp. $F$-split) for a dense set of $p$. Projective varieties $X$, which are globally F-regular type, have remarkable geometric and cohomological properties: (1) X must be normal, Cohen-Macaulay with rational singularities, and must have log terminal singularities if it is $\mathbb{Q}$-Gorenstein; $(2) H^{i}(X, \mathcal{L})=0$ for $i>0$ and nef line bundle $\mathcal{L}$.

Let $\mathcal{U}_{C, \omega}$ be moduli spaces of semistable parabolic bundles of rank $r$ and degree $d$ on smooth curves $C$ of genus $g \geq 0$ with parabolic structures determined by $\omega=\left(k,\{\vec{n}(x), \vec{a}(x)\}_{x \in I}\right)$ and

$$
\operatorname{det}: \mathcal{U}_{C, \omega} \rightarrow J_{C}^{d}
$$

be the determinant morphism. For any $L \in J_{C}^{d}$, the fiber

$$
\mathcal{U}_{C, \omega}^{L}:=\operatorname{det}^{-1}(L)
$$

is called moduli space of semistable parabolic bundles with a fixed determinant $L$. Then the first main result in this article is

Theorem 1.1 (See Theorem 3.7). The moduli spaces $\mathcal{U}_{C, \omega}^{L}$ are of globally F-regular type.

When the projective curve $C$ has exactly one node (irreducible, or reducible), the moduli space $\mathcal{U}_{C, \omega}$ is not normal and its normalization is a moduli space $\mathcal{P}_{\omega}$ of semistable generalized parabolic sheaves (GPS) on $\widetilde{C}$ (where $\widetilde{C}$ is normalization of $C$ ). There exist a similar determinant morphism det $: \mathcal{P}_{\omega} \rightarrow J_{\widetilde{C}}^{d}$. For any $L \in J_{\widetilde{C}}^{d}$, the fiber

$$
\mathcal{P}_{\omega}^{L}:=\operatorname{det}^{-1}(L)
$$

is called a moduli space of semistable generalized parabolic sheaves (GPS) with a fixed determinant $L$ on $\widetilde{C}$. Then the second main result in this article is

Theorem 1.2 (See Theorem4.7 and Theorem4.15). The moduli spaces $\mathcal{P}_{\omega}^{L}$ are of globally F-regular type.

To describe the idea of proof, recall that the moduli space $\mathcal{U}_{C, \omega}^{L}$ is a GIT quotient $\left(\mathcal{R}_{\omega}^{s s}\right)^{L} / / \mathrm{SL}(V)$, where $\left(\mathcal{R}_{\omega}^{s s}\right)^{L} \subset \mathcal{R}_{F}^{L}$ is a open set of a quasi-projective variety $\mathcal{R}_{F}^{L}$ ( i.e. the set of GIT semistable points respect to a polarization $\Theta_{\mathcal{R}, \omega}$ determined by $\omega$ ). Then our idea is to find a flag bundle $\mathcal{R}_{F}^{\prime L} \stackrel{\hat{f}}{\rightarrow} \mathcal{R}_{F}^{L}$ over $\mathcal{R}_{F}^{L}$ and a data $\omega^{\prime}$ such that 
$\mathcal{U}_{C, \omega^{\prime}}^{L}=\left(\mathcal{R}_{\omega^{\prime}}^{\prime s s}\right)^{L} / / \mathrm{SL}(V)$ is a Fano variety with an open subvariety $X \subset \mathcal{U}_{C, \omega^{\prime}}^{L}$ and a morphism $X \stackrel{f}{\rightarrow} \mathcal{U}_{C, \omega}^{L}$ satisfying $f_{*} \mathcal{O}_{X}=\mathcal{O}_{\mathcal{U}_{C, \omega}^{L}}$. Since Fano varieties are globally $F$-regular type by Proposition 6.3 of [15], so are $X$ and $\mathcal{U}_{C, \omega}^{L}$ if the equality $f_{*} \mathcal{O}_{X}=\mathcal{O}_{\mathcal{U}_{C, \omega}^{L}}$ commutes with modulo $p$ reductions for a dense set of $p$. To prove that $f_{*} \mathcal{O}_{X}=\mathcal{O}_{\mathcal{U}_{C, \omega}^{L}}$ commutes with modulo $p$ reductions for a dense set of $p$, one has to show in particular that a GIT quotient over $\mathbb{Z}$ must commute with modulo $p$ reductions for a dense set of $p$, which is Lemma 2.9 (we thought at first that Lemma 2.9 must be well-known to experts, but we are not able to find any reference). We formulate our idea in Proposition 2.10 in a general setting, the proof of Theorem 1.1 and Theorem 1.2 becomes to check conditions in Proposition 2.10.

We describe briefly content of the article. In Section 2, we collect notions and properties of globally $F$-regular type varieties, in particular, we formulate and prove Proposition 2.10 , which is our technical tool to show globally F-regular type of GIT quotients. In Section 3, we recall some facts about moduli spaces of parabolic bundles and prove Theorem 1.1. Finally, we prove Theorem 1.2 in Section 4.

Acknowledegements: Xiaotao Sun would like to thank C. S. Seshadri for a number of emails of discussions about Lemma 2.9, and he also would like to thank K. Schwede and K. E. Smith for discussions (by emails) of globally $F$-regular type varieties.

\section{Globally F-Regular varieties}

We collect firstly some notions and facts of globally F-regular varieties over a perfect field $k$ of positive characteristic and recall the definition of globally F-regular type of varieties over a field of characteristic zero. Our main references here are [1], 13] and [15].

Let $X$ be a variety over a perfect field $k$ of $\operatorname{char}(k)=p>0$,

$$
F: X \rightarrow X
$$

be the Frobenius map and $F^{e}: X \rightarrow X$ be the e-th iterate of Frobenius map. When $X$ is normal, for any (weil) divisor $D \in \operatorname{Div}(X)$,

$$
\mathcal{O}_{X}(D)(V)=\left\{f \in K(X)\left|\operatorname{div}_{V}(f)+D\right|_{V} \geq 0\right\}, \quad \forall V \subset X
$$

is a reflexive subsheaf of constant sheaf $K=K(X)$. In fact, we have

$$
\mathcal{O}_{X}(D)=j_{*} \mathcal{O}_{X s m}(D)
$$

where $j: X^{s m .} \hookrightarrow X$ is the open set of smooth points, and $\mathcal{O}_{X}(D)$ is an invertible sheaf if and only if $D$ is a Cartier divisor. 
Definition 2.1. A normal variety $X$ over a perfect field is called stably Frobenius $D$-split if $\mathcal{O}_{X} \rightarrow F_{*}^{e} \mathcal{O}_{X}(D)$ is split for some $e>0$. $X$ is called globally F-regular if $X$ is stably Frobenius $D$-split for any effective divisor $D$.

The advantage of this definition is that any open set $U \subset X$ of a globally F-regular variety $X$ is globally $\mathrm{F}$-regular. Its disadvantage is the requirement of normality of $X$. When $X$ is not normal, one possible remedy of Definition 2.1 is to require that $D$ is a Cartier divisor. Then it loses the advantage that any open set $U \subset X$ is globally F-regular since a Cartier divisor on $U$ may not be extended to a Cartier divisor on $X$. But, when $X$ is a projective variety and is stably Frobenius $D$-split for any effective Cartier $D, X$ must be normal and Cohen-Macaulay according to K. E. Smith (Theorem 3.10 and Theorem 4.1 of [15]).

Proposition 2.2 (Theorem 3.10 of [15]). Let $X$ be a projective variety over a perfect field. Then the following statements are equivalent.

(1) $X$ is normal and is stably Frobenius D-split for any effective D;

(2) $X$ is stably Frobenius D-split for any effective Cartier D;

(3) For any ample line bundle $\mathcal{L}$, the section ring of $X$

$$
R(X, \mathcal{L})=\bigoplus_{n=0}^{\infty} H^{0}\left(X, \mathcal{L}^{n}\right)
$$

is strongly F-regular.

Proof. It is clear that $(1) \Rightarrow(2)$, and $(2) \Rightarrow(3)$ is proved in Theorem 3.10 of [15]. That $(3) \Rightarrow(1)$ is a modification of the proof in [15]. By Theorem 4.1 of [15], $X$ is normal and Cohen-Macaulay. Let $X^{s m} \cdot \subset X$ be the open set of smooth points, then $R(X, \mathcal{L})=R\left(X^{s m}, \mathcal{L}\right)$ and, for any effective $D \in \operatorname{Div}(X), D \cap X^{s m}$. is an effective Cartier divisor on $X^{s m}$. Then the proof of $(1) \Rightarrow(3)$ in Theorem 3.10 of [15] implies that $X^{s m}$. is stably Frobenius $D \cap X^{s m}$.split, which implies that $X$ is stably Frobenius $D$-split.

A variety $X$ is called $F$-split if $\mathcal{O}_{X} \rightarrow F_{*} \mathcal{O}_{X}$ is split. In particular, globally F-regular varieties are $F$-split. Let $X \stackrel{f}{\rightarrow} Y$ be a morphism such that $f_{*} \mathcal{O}_{X}=\mathcal{O}_{Y}$, then any splitting map $F_{*} \mathcal{O}_{X} \stackrel{\psi}{\rightarrow} \mathcal{O}_{X}$ of $\mathcal{O}_{X} \rightarrow F_{*} \mathcal{O}_{X}$ induces a splitting map $F_{*} \mathcal{O}_{Y}=F_{*} f_{*} \mathcal{O}_{X}=f_{*} F_{*} \mathcal{O}_{X} \stackrel{f_{*} \psi}{\longrightarrow} f_{*} \mathcal{O}_{X}=\mathcal{O}_{Y}$. There is a generalization of above useful observation.

Lemma 2.3 (Corollary 6.4 of [13]). Let $f: X \rightarrow Y$ be a morphism of varieties over a perfect field $k$ of $\operatorname{char}(k)=p>0$. If the natural map $\mathcal{O}_{Y} \stackrel{i}{\rightarrow} f_{*} \mathcal{O}_{X}$ splits and $X$ is globally F-regular, then $Y$ is stably 
Frobenius D-split for any effective Cartier divisor D, and it is globally F-regular when $Y$ is normal.

Proof. For any Cartier divisor $D \in \operatorname{Div}(Y)$ defined by a section $s \in$ $\Gamma\left(Y, \mathcal{O}_{Y}(D)\right)$, let $H=f^{*} D$ and $F_{*}^{e} \mathcal{O}_{X}(H) \stackrel{h}{\rightarrow} \mathcal{O}_{X}$ be a splitting of $\mathcal{O}_{X} \rightarrow F_{*}^{e} \mathcal{O}_{X} \stackrel{F_{*}^{e} f^{*}(s)}{\longrightarrow} F_{*}^{e} \mathcal{O}_{X}(H)$, and $f_{*} \mathcal{O}_{X} \stackrel{j}{\rightarrow} \mathcal{O}_{Y}$ be a splitting of $\mathcal{O}_{Y} \stackrel{i}{\rightarrow} f_{*} \mathcal{O}_{X}$. Then $\mathcal{O}_{Y}(D) \stackrel{1 \otimes i}{\longrightarrow} \mathcal{O}_{Y}(D) \otimes f_{*} \mathcal{O}_{X}=f_{*} \mathcal{O}_{X}(H)$ induces

$$
F_{*}^{e} \mathcal{O}_{Y}(D) \stackrel{F_{*}^{e} 1 \otimes i}{\longrightarrow} F_{*}^{e} f_{*} \mathcal{O}_{X}(H)=f_{*} F_{*}^{e} \mathcal{O}_{X}(H) \stackrel{f_{*} h}{\longrightarrow} f_{*} \mathcal{O}_{X} \stackrel{j}{\rightarrow} \mathcal{O}_{Y}
$$

is a splitting of $\mathcal{O}_{Y} \rightarrow F_{*}^{e} \mathcal{O}_{Y} \stackrel{F_{*}^{e} s}{\longrightarrow} F_{*}^{e} \mathcal{O}_{Y}(D)$. When $Y$ is normal, let $Y_{0} \subset Y$ be the open set of smooth points, $Y$ is globally F-regular if and only if $Y_{0}$ is stably Frobenius $D$-split for any effective Cartier divisor $D \in \operatorname{Div}\left(Y_{0}\right)$, which is true by applying above argument to $f^{-1}\left(Y_{0}\right) \stackrel{f}{\rightarrow} Y_{0}$.

For any scheme $X$ of finite type over a field $K$ of characteristic zero, there is a finitely generated $\mathbb{Z}$-algebra $A \subset K$ and an $A$-flat scheme

$$
X_{A} \rightarrow S=\operatorname{Spec}(A)
$$

such that $X_{K}=X_{A} \times{ }_{S} \operatorname{Spec}(K) \cong X . X_{A} \rightarrow S=\operatorname{Spec}(A)$ is called an integral model of $X / K$, and a closed fiber $X_{s}=X_{A} \times_{S} \operatorname{Spec}(\overline{k(s)})$ is called "modulo $p$ reduction of $X$ " where $p=\operatorname{char}(k(s))>0$.

Definition 2.4. A variety $X$ over a field of characteristic zero is said to be of globally F-regular type (resp. F-split type) if its "modulo $p$ reduction of $X$ " are globally F-regular (resp. F-split) for a dense set of $p$.

Projective varieties of globally F-regular type have many nice properties and a good vanishing theorem of cohomology.

Theorem 2.5 (Corollary 5.3 and Corollary 5.5 of [15]). Let $X$ be a projective variety over a field of characteristic zero. If $X$ is of globally F-regular type, then we have

(1) $X$ is normal, Cohen-Macaulay with rational singularities. If $X$ is $\mathbb{Q}$-Gorenstein, then $X$ has log terminal singularities.

(2) For any nef line bundle $\mathcal{L}$ on $X$, we have $H^{i}(X, \mathcal{L})=0$ when $i>0$. In particular, $H^{i}\left(X, \mathcal{O}_{X}\right)=0$ whenever $i>0$.

A normal projective variety $X$ is called a Fano variety if

$$
\omega_{X}^{-1}=\mathcal{H o m}_{\mathcal{O}_{X}}\left(\omega_{X}, \mathcal{O}_{X}\right)
$$

is an ample line bundle. One of important examples of globally $F$ regular type varieties is 
Proposition 2.6. (15, Proposition 6.3]) A Fano variety (over a field of characteristic zero) with at most rational singularities is of globally F-regular type.

To show that a variety $Y$ is of globally F-regular type, one possible approach is to construct an open set $X$ of a Fano variety (thus $X$ is of globally F-regular type) with a morphism $f: X \rightarrow Y$ such that $f_{*} \mathcal{O}_{X}=\mathcal{O}_{Y}$. Then $Y$ is of globally F-regular type if the following characteristic zero analogy of Lemma 2.3 is true.

Question 2.7. Let $X \stackrel{f}{\rightarrow} Y$ be a morphism of varieties over a field $K$ of $\operatorname{char}(K)=0$ such that $\mathcal{O}_{Y} \rightarrow f_{*} \mathcal{O}_{X}$ is split and $X$ is of globally F-regular type. Is $Y$ a variety of globally F-regular type?

Let $f_{*} \mathcal{O}_{X} \stackrel{\beta}{\rightarrow} \mathcal{O}_{Y}$ be a splitting of $\mathcal{O}_{Y} \rightarrow f_{*} \mathcal{O}_{X}$. Then Question 2.7 consists: (1) Can we choose a model $f_{A}: X_{A} \rightarrow Y_{A}$ of $f: X \rightarrow Y$ such that the $\mathcal{O}_{Y}$-homomorphism $\left(f_{A_{*}} \mathcal{O}_{X_{A}}\right) \otimes_{A} K \stackrel{\beta}{\rightarrow} \mathcal{O}_{Y_{A}} \otimes_{A} K$ can be extended to $f_{A_{*}} \mathcal{O}_{X_{A}} \stackrel{\beta_{A}}{\longrightarrow} \mathcal{O}_{Y_{A}}$ ? (2) Is there a dense set of closed point $\operatorname{Spec}(\overline{k(s)}) \rightarrow S=\operatorname{Spec}(A)$ such that $i_{s}^{*} f_{A *} \mathcal{O}_{X_{A}}=f_{s *} j_{s}^{*} \mathcal{O}_{X_{A}}$ ? where $Y_{s}=Y_{A} \times_{A} \overline{k(s)} \stackrel{i_{s}}{\rightarrow} Y_{A}, X_{s}=X_{A} \times_{A} \overline{k(s)} \stackrel{j_{s}}{\rightarrow} X_{A}$ and

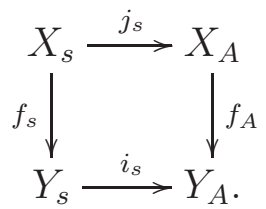

Definition 2.8. A morphism $X \stackrel{f}{\rightarrow} Y$ of varieties over a field $K$ of $\operatorname{char}(K)=0$ is called $p$-compatible if there is an integral model $X_{A} \stackrel{f_{A}}{\longrightarrow}$ $Y_{A}$ such that $i_{s}^{*} f_{A *} \mathcal{O}_{X_{A}}=f_{s *} j_{s}^{*} \mathcal{O}_{X_{A}}$ for $s \in \operatorname{Spec}(A)$.

It is clear that (1) has an affirmative answer when either $f_{*} \mathcal{O}_{X}$ is a coherent $\mathcal{O}_{Y}$-module or the splitting map $\beta: f_{*} \mathcal{O}_{X} \rightarrow \mathcal{O}_{Y}$ is a homomorphism of $\mathcal{O}_{Y}$-algebras. (2) has an affirmative answer for flat morphism $f: X \rightarrow Y$ with coherent $R^{i} f_{*} \mathcal{O}_{X}(i \geq 0)$. It is also clear that any affine morphism must be $p$-compatible. When $X, Y$ are open set of GIT quotients and $f: X \rightarrow Y$ is induced by a $G$-invariant $p$ compatible morphism $\hat{f}: \mathcal{R}^{\prime} \rightarrow \mathcal{R}$ of parameter spaces, we will show that $f: X \rightarrow Y$ is $p$-compatible morphism in Proposition 2.10, which will need the following lemma.

Lemma 2.9. Let $X \rightarrow S=\operatorname{Spec}(A)$ be a flat projective morphism, $A$ be an integral $\mathbb{Z}$-algebra of finite type and $G \rightarrow S$ be a $S$-flat reductive 
group scheme with action on $X$ over $S$. If $L$ is a relative ample line bundle on $X$ linearizing the action of $G$, let

$$
X^{s s}(L) \stackrel{\pi}{\rightarrow} X^{s s}(L) / / G:=Y
$$

be the GIT quotient over $S$. Assume that the geometrically generic fiber of $X^{s s}(L) \rightarrow S$ is an irreducible normal variety. Then there is a dense open set $U \subset S$ such that for any $s \in U$

$$
Y \times_{S} \overline{k(s)} \cong X_{s}^{s s}\left(L_{s}\right) / / G_{s}
$$

where $X_{s}=X \times_{S} \overline{k(s)}$ (resp. $G_{s}=G \times_{S} \overline{k(s)}$ ) is the geomerically closed fiber of $X \rightarrow S$ (resp. $G \rightarrow S$ ) at $\operatorname{Spec}(\overline{k(s))} \rightarrow S$.

Proof. Let $X^{s s}(L) \times_{S} \overline{k(s)} \stackrel{\pi_{s}}{\rightarrow} Y_{s}$ be the pullback of $X^{s s}(L) \stackrel{\pi}{\rightarrow} Y$ under the base change $\operatorname{Spec}(\overline{k(s))} \rightarrow S$. By Proposition 7 of [14],

$$
X^{s s}(L) \times{ }_{S} \overline{k(s)}=X_{s}^{s s}\left(L_{s}\right) .
$$

Then there is a unique $\overline{k(s)}$-morphism $X_{s}^{s s}\left(L_{s}\right) / / G_{s} \stackrel{\theta}{\rightarrow} Y_{s}$ such that

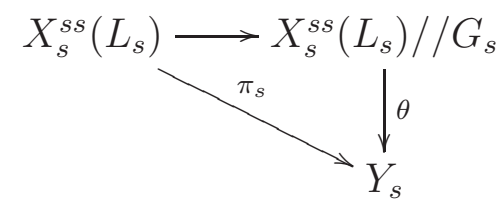

is commutative. Let $\overline{Y_{s}}:=X_{s}^{s s}\left(L_{s}\right) / / G_{s}, k=\overline{k(s)}$, it is known that $\theta$ induces a bijective map $\overline{Y_{s}}(k) \stackrel{\theta}{\rightarrow} Y_{s}(k)$ on the sets of $k$-points (cf. Proposition 9 (i) of [14]). By the assumption, geometrically generic fiber of $Y \rightarrow S$ is an irreducible normal projective variety. Thus there is a dense open set $U \subset S$ such that any closed point $\operatorname{Spec}(\overline{k(s))} \rightarrow U$ satisfies (1) $Y_{s}$ is normal, and (2) the morphism $X_{s}^{s s}\left(L_{s}\right) \stackrel{\pi_{s}}{\longrightarrow} Y_{s}$ is generic smooth, where (1) is (iv) of Théoréme (12.2.4) in [2] and (2) holds since $K=Q(A)=k(S)$ is a field of characteristic zero. Then generic smoothness of $\pi_{s}$ implies the generic smoothness of $\overline{Y_{s}} \stackrel{\theta}{\rightarrow} Y_{s}$, which must be an isomorphism by Zariski main theorem since $Y_{s}$ is normal.

Let $(\hat{Y}, L),\left(\hat{Z}, L^{\prime}\right)$ be polarized projective varieties over an algebraically closed field $K$ of characteristic zero with actions of a reductive group scheme $G$ over $K$, and $\hat{Y}^{s s}(L) \subset \hat{Y}$ (resp. $\left.\hat{Y}^{s}(L) \subset \hat{Y}^{s s}(L)\right)$ be the open set of GIT semi-stable (resp. GIT stable) points of $\hat{Y}$. Then there are projective GIT quotients

$$
\hat{Y}^{s s}(L) \stackrel{\psi}{\rightarrow} Y:=\hat{Y}^{s s}(L) / / G, \quad \hat{Z}^{s s}\left(L^{\prime}\right) \stackrel{\varphi}{\rightarrow} Z:=\hat{Z}^{s s}\left(L^{\prime}\right) / / G .
$$

Proposition 2.10. Let Z, Y be the GIT quotients in (2.1). Assume 
(1) there are $G$-invariant normal open subschemes $\mathcal{R} \subset \hat{Y}, \mathcal{R}^{\prime} \subset \hat{Z}$ such that $\hat{Y}^{s s}(L) \subset \mathcal{R}, Z^{s s}\left(L^{\prime}\right) \subset \mathcal{R}^{\prime}$;

(2) there is a G-invariant p-compatible morphism $\mathcal{R}^{\prime} \stackrel{\hat{f}}{\rightarrow} \mathcal{R}$ such that $\hat{f}_{*} \mathcal{O}_{\mathcal{R}^{\prime}}=\mathcal{O}_{\mathcal{R}}$

(3) there is an $G$-invariant open set $W \subset Z^{s s}\left(L^{\prime}\right)$ such that

$$
\operatorname{Codim}\left(\mathcal{R}^{\prime} \backslash W\right) \geq 2, \quad \hat{X}=\varphi^{-1} \varphi(\hat{X})
$$

where $\hat{X}=W \cap \hat{f}^{-1}\left(\hat{Y}^{s s}(L)\right)$.

If $Z$ is of globally F-regular type. Then so is $Y$.

Proof. Let $X=\varphi(\hat{X}) \subset Z$, which is an open set of $Z$ since

$$
\varphi\left(Z^{s s}\left(L^{\prime}\right) \backslash \hat{X}\right)=Z \backslash X
$$

by the condition $\varphi^{-1}(X)=\hat{X}$ and that $Z^{s s}\left(L^{\prime}\right) \backslash \hat{X}$ is a $G$-invariant closed subset. There is a morphism $X \stackrel{f}{\rightarrow} Y$ such that

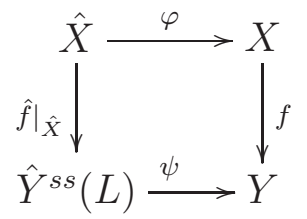

is commutative. For any open set $U \subset Y$, since $\hat{f}_{*} \mathcal{O}_{\mathcal{R}^{\prime}}=\mathcal{O}_{\mathcal{R}}$, we have

$$
\begin{aligned}
\mathcal{O}_{Y}(U) & =\mathcal{O}_{\mathcal{R}}\left(\psi^{-1}(U)\right)^{i n v .}=\mathcal{O}_{\mathcal{R}^{\prime}}\left(\hat{f}^{-1} \psi^{-1}(U)\right)^{i n v .} \\
& =\mathcal{O}_{\mathcal{R}^{\prime}}\left(W \cap \hat{f}^{-1} \psi^{-1}(U)\right)^{i n v .}=\mathcal{O}_{\hat{X}}\left(\left.\hat{f}\right|_{\hat{X}} ^{-1} \psi^{-1}(U)\right)^{i n v} \\
& =\mathcal{O}_{\hat{X}}\left(\varphi^{-1} f^{-1}(U)\right)^{i n v .}=\mathcal{O}_{X}\left(f^{-1}(U)\right)=f_{*} \mathcal{O}_{X}(U)
\end{aligned}
$$

where the third equality holds because $\hat{f}^{-1} \psi^{-1}(U) \backslash W \cap \hat{f}^{-1} \psi^{-1}(U)=$ $\hat{f}^{-1} \psi^{-1}(U) \cap\left(\mathcal{R}^{\prime} \backslash W\right)$ has codimension at least two. Thus we have

$$
\mathcal{O}_{Y}=f_{*} \mathcal{O}_{X} \text {, where } X \text { is of globally F-regular type. }
$$

To show that $Y$ is of globally F-regular type, it is enough to show that the morphism $X \stackrel{f}{\rightarrow} Y$ is $p$-compatible.

Let $\left(\hat{Y}_{A}, \mathcal{L}\right),\left(\hat{Z}_{A}, \mathcal{L}^{\prime}\right)$ be integral models of $(\hat{Y}, L),\left(\hat{Z}, L^{\prime}\right)$ with actions of a reductive group scheme $G_{A}$ over $S=\operatorname{Spec}(A)$, and $\hat{Y}_{A}^{s s}(\mathcal{L}) \subset \hat{Y}_{A}$ (resp. $\hat{Y}_{A}^{s}(\mathcal{L}) \subset \hat{Y}_{A}^{s s}(\mathcal{L})$ ) be the open subscheme of GIT semi-stable (resp. GIT stable) points of $\hat{Y}_{A}$. Then there are GIT quotients

$$
\hat{Y}_{A}^{s s}(\mathcal{L}) \stackrel{\psi_{A}}{\longrightarrow} Y_{A}:=\hat{Y}_{A}^{s s}(\mathcal{L}) / / G_{A}, \quad \hat{Z}_{A}^{s s}\left(\mathcal{L}^{\prime}\right) \stackrel{\varphi_{A}}{\longrightarrow} Z_{A}:=\hat{Z}_{A}^{s s}\left(\mathcal{L}^{\prime}\right) / / G_{A},
$$

which are projective over $S=\operatorname{Spec}(A)$ and $\psi_{A}, \varphi_{A}$ are surjective $G_{A^{-}}$ invariant affine morphisms (cf. Theorem 4 of [14]). 
We can choose $G_{A}$-invariant open subschemes $\mathcal{R}_{A} \subset \hat{Y}_{A}, \mathcal{R}_{A}^{\prime} \subset \hat{Z}_{A}$, $W_{A} \subset Z_{A}^{s s}\left(\mathcal{L}^{\prime}\right), X_{A} \subset Z_{A}$ and a $G_{A^{-}}$invariant morphism $\mathcal{R}_{A}^{\prime} \stackrel{\hat{f}_{A}}{\longrightarrow} \mathcal{R}_{A}$ such that $\hat{Y}_{A}^{s s}(\mathcal{L}) \subset \mathcal{R}_{A}, Z_{A}^{s s}\left(\mathcal{L}^{\prime}\right) \subset \mathcal{R}_{A}^{\prime}, \hat{f}_{A *} \mathcal{O}_{\mathcal{R}_{A}^{\prime}}=\mathcal{O}_{\mathcal{R}_{A}}$. Let

$$
\hat{X}_{A}=\varphi_{A}^{-1}\left(X_{A}\right), \mathcal{R}_{s}^{\prime}=\mathcal{R}_{A}^{\prime} \times_{A} \overline{k(s)}, \mathcal{R}_{s}=\mathcal{R}_{A} \times_{A} \overline{k(s)},
$$

and $\hat{f}_{s}=\hat{f}_{A} \otimes \overline{k(s)}(\forall s \in S)$. Then we have $\hat{f}_{s *} \mathcal{O}_{\mathcal{R}_{s}^{\prime}}=\mathcal{O}_{\mathcal{R}_{s}}$,

$$
\operatorname{Codim}\left(\mathcal{R}_{s}^{\prime} \backslash W_{s}\right) \geq 2, \hat{X}_{s}=W_{s} \cap \hat{f}_{s}^{-1}\left(\hat{Y}_{A}^{s s}(\mathcal{L}) \times_{A} \overline{k(s)}\right)
$$

(by shrinking $S$ ) where $W_{s}=W_{A} \times_{A} \overline{k(s)}, \hat{X}_{s}=\hat{X}_{A} \times_{A} \overline{k(s)}$ and

$$
\hat{Y}_{A}^{s s}(\mathcal{L}) \times_{A} \overline{k(s)}=\hat{Y}_{s}^{s s}\left(\mathcal{L}_{s}\right), \quad \hat{Z}_{A}^{s s}\left(\mathcal{L}^{\prime}\right) \times_{A} \overline{k(s)}=\hat{Z}_{s}^{s s}\left(\mathcal{L}_{s}^{\prime}\right)
$$

(cf. Proposition 7 of [14]). Then, by Lemma 2.9, we have

$$
Z_{s}=Z_{s}^{s s}\left(\mathcal{L}_{s}^{\prime}\right) / / G_{s}, \quad Y_{s}=Y_{s}^{s s}\left(\mathcal{L}_{s}\right) / / G_{s}
$$

Thus, for any open sets $U \subset Z_{s}, V \subset Y_{s}$, one has

$$
\mathcal{O}_{Z_{s}}(U)=\mathcal{O}_{\mathcal{R}_{s}^{\prime}}\left(\varphi_{s}^{-1}(U)\right)^{i n v .}, \quad \mathcal{O}_{Y_{s}}(V)=\mathcal{O}_{\mathcal{R}_{s}}\left(\psi_{s}^{-1}(V)\right)^{i n v .}
$$

Recall $X_{s} \subset Z_{s}, \varphi_{s}^{-1}\left(X_{s}\right)=\hat{X}_{s}=W_{s} \cap \hat{f}_{s}^{-1}\left(\hat{Y}_{s}^{s s}\left(\mathcal{L}_{s}\right)\right)$ and consider

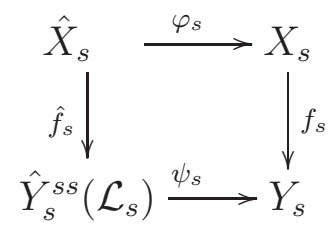

we have $\mathcal{O}_{Y_{s}}(V)=\mathcal{O}_{\mathcal{R}_{s}}\left(\psi_{s}^{-1}(V)\right)^{i n v}=\mathcal{O}_{\mathcal{R}_{s}^{\prime}}\left(\hat{f}_{s}^{-1}\left(\psi_{s}^{-1}(V)\right)\right)^{i n v}$ since $\hat{f}_{s *} \mathcal{O}_{\mathcal{R}_{s}^{\prime}}=\mathcal{O}_{\mathcal{R}_{s}}$. Because the codimension of

$$
\hat{f}_{s}^{-1}\left(\psi_{s}^{-1}(V)\right) \backslash W_{s} \cap \hat{f}_{s}^{-1}\left(\psi_{s}^{-1}(V)\right)=\hat{f}_{s}^{-1}\left(\psi_{s}^{-1}(V)\right) \cap\left(\mathcal{R}_{s}^{\prime} \backslash W_{s}\right)
$$

is at least two, we have

$$
\begin{aligned}
\mathcal{O}_{Y_{s}}(V) & =\mathcal{O}_{\hat{X}_{s}}\left(\hat{f}_{s}^{-1}\left(\psi_{s}^{-1}(V)\right)\right)^{i n v .}=\mathcal{O}_{\hat{X}_{s}}\left(\varphi_{s}^{-1} f_{s}^{-1}(V)\right)^{i n v} \\
& =\mathcal{O}_{X_{s}}\left(f_{s}^{-1}(V)\right)=\left(f_{s}\right)_{*} \mathcal{O}_{X_{s}}(V) .
\end{aligned}
$$

Thus $\mathcal{O}_{Y_{s}}=\left(f_{s}\right)_{*} \mathcal{O}_{X_{s}}$, which implies that $f: X \rightarrow Y$ is $p$-compatible and $Y$ is of globally F-regular type since $X$ is so. 


\section{Globally F-Regular type of Moduli spaces of PARABOLIC BUNDLES}

In this section, we prove that moduli spaces of parabolic bundles with a fixed determinant on a smooth curve are of globally F-regular type.

Let $C$ be an irreducible projective curve of genus $g \geq 0$ over an algebraically closed field $K$ of characteristic zero, which has at most one node $x_{0} \in C$. Let $I$ be a finite set of smooth points of $C$, and $E$ be a coherent sheaf of rank $r$ and degree $d$ on $C$ (the rank $r(E)$ is defined to be dimension of $E_{\xi}$ at generic point $\xi \in C$, and $d=\chi(E)-r(1-g)$ ).

Definition 3.1. By a quasi-parabolic structure of $E$ at a smooth point $x \in C$, we mean a choice of flag of quotients

$$
E_{x}=Q_{l_{x}+1}(E)_{x} \rightarrow Q_{l_{x}}(E)_{x} \rightarrow \cdots \cdots \rightarrow Q_{1}(E)_{x} \rightarrow Q_{0}(E)_{x}=0
$$

of the fibre $E_{x}, n_{i}(x)=\operatorname{dim}\left(\operatorname{ker}\left\{Q_{i}(E)_{x} \rightarrow Q_{i-1}(E)_{x}\right\}\right)\left(1 \leq i \leq l_{x}+1\right)$ are called type of the flags. If, in addition, a sequence of integers

$$
0 \leq a_{1}(x)<a_{2}(x)<\cdots<a_{l_{x}+1}(x)<k
$$

are given, we call that $E$ has a parabolic structure of type

$$
\vec{n}(x)=\left(n_{1}(x), n_{2}(x), \cdots, n_{l_{x}+1}(x)\right)
$$

and weight $\vec{a}(x)=\left(a_{1}(x), a_{2}(x), \cdots, a_{l_{x}+1}(x)\right)$ at $x \in C$.

Definition 3.2. For any subsheaf $F \subset E$, let $Q_{i}(E)_{x}^{F} \subset Q_{i}(E)_{x}$ be the image of $F$ and $n_{i}^{F}=\operatorname{dim}\left(k e r\left\{Q_{i}(E)_{x}^{F} \rightarrow Q_{i-1}(E)_{x}^{F}\right\}\right)$. Let

$$
\begin{aligned}
& \operatorname{par} \chi(E):=\chi(E)+\frac{1}{k} \sum_{x \in I} \sum_{i=1}^{l_{x}+1} a_{i}(x) n_{i}(x), \\
& \operatorname{par} \chi(F):=\chi(F)+\frac{1}{k} \sum_{x \in I} \sum_{i=1}^{l_{x}+1} a_{i}(x) n_{i}^{F}(x) .
\end{aligned}
$$

Then $E$ is called semistable (resp., stable) for $\omega=\left(k,\{\vec{n}(x), \vec{a}(x)\}_{x \in I}\right)$ if for any nontrivial $E^{\prime} \subset E$ such that $E / E^{\prime}$ is torsion free, one has

$$
\operatorname{par} \chi\left(E^{\prime}\right) \leq \frac{\operatorname{par} \chi(E)}{r} \cdot r\left(E^{\prime}\right)(\text { resp., }<) .
$$

Theorem 3.3 (Theorem X1 of [10] or Theorem 2.13 of [18] for arbitrary rank). There exists a seminormal projective variety

$$
\mathcal{U}_{C, \omega}:=\mathcal{U}_{C}\left(r, d,\{k, \vec{n}(x), \vec{a}(x)\}_{x \in I}\right),
$$

which is the coarse moduli space of s-equivalence classes of semistable parabolic sheaves $E$ of rank $r$ and $\chi(E)=\chi=d+r(1-g)$ with parabolic 
structures of type $\{\vec{n}(x)\}_{x \in I}$ and weights $\{\vec{a}(x)\}_{x \in I}$ at points $\{x\}_{x \in I}$. If $C$ is smooth, then it is normal, with only rational singularities.

Recall the construction of $\mathcal{U}_{C, \omega}=\mathcal{U}_{C}(r, d, \omega)$. Fix a line bundle $\mathcal{O}(1)=\mathcal{O}_{C}(c \cdot y)$ on $C$ of $\operatorname{deg}(\mathcal{O}(1))=c$, let $\chi=d+r(1-g), P$ denote the polynomial $P(m)=c r m+\chi, \mathcal{O}_{C}(-N)=\mathcal{O}(1)^{-N}$ and $V=\mathbb{C}^{P(N)}$. Let $\mathrm{Q}$ be the Quot scheme of quotients $V \otimes \mathcal{O}_{C}(-N) \rightarrow F \rightarrow 0$ (of rank $r$ and degree $d$ ) on $C$. Thus there is on $C \times \mathbf{Q}$ a universal quotient

$$
V \otimes \mathcal{O}_{C \times \mathbf{Q}}(-N) \rightarrow \mathcal{F} \rightarrow 0 .
$$

Let $\mathcal{F}_{x}=\left.\mathcal{F}\right|_{\{x\} \times \mathbf{Q}}$ and $\operatorname{Flag}_{\vec{n}(x)}\left(\mathcal{F}_{x}\right) \rightarrow \mathbf{Q}$ be the relative flag scheme of type $\vec{n}(x)$. Let

$$
\mathcal{R}=\underset{x \in I}{\times_{\mathbf{Q}}} F \operatorname{lag}_{\vec{n}(x)}\left(\mathcal{F}_{x}\right) \rightarrow \mathbf{Q},
$$

on which reductive group $\mathrm{SL}(V)$ acts. The data $\omega=\left(k,\{\vec{n}(x), \vec{a}(x)\}_{x \in I}\right)$, more precisely, the weight $\left(k,\{\vec{a}(x)\}_{x \in I}\right)$ determines a polarisation

$$
\Theta_{\mathcal{R}, \omega}=\left(\operatorname{det} R \pi_{\mathcal{R}} \mathcal{E}\right)^{-k} \otimes \bigotimes_{x \in I}\left\{\bigotimes_{i=1}^{l_{x}} \operatorname{det}\left(\mathcal{Q}_{\{x\} \times \mathcal{R}, i}\right)^{d_{i}(x)}\right\} \otimes \bigotimes_{q} \operatorname{det}\left(\mathcal{E}_{y}\right)^{\ell}
$$

on $\mathcal{R}$ such that the open set $\mathcal{R}_{\omega}^{s s}$ (resp. $\mathcal{R}_{\omega}^{s}$ ) of GIT semistable (resp. GIT stable) points are precisely the set of semistable (resp. stable) parabolic sheaves on $C$ (see [18]), where $\mathcal{E}$ is the pullback of $\mathcal{F}$ (under $C \times \mathcal{R} \rightarrow C \times \mathbf{Q}), \operatorname{det} R \pi_{\mathcal{R}} \mathcal{E}$ is determinant line bundle of cohomology,

$$
\mathcal{E}_{x}=\mathcal{Q}_{\{x\} \times \mathcal{R}, l_{x}+1} \rightarrow \mathcal{Q}_{\{x\} \times \mathcal{R}, l_{x}} \rightarrow \mathcal{Q}_{\{x\} \times \mathcal{R}, l_{x}-1} \rightarrow \cdots \rightarrow \mathcal{Q}_{\{x\} \times \mathcal{R}, 1} \rightarrow 0
$$

are universal quotients on $\mathcal{R}$ of type $\vec{n}(x), d_{i}(x)=a_{i+1}(x)-a_{i}(x)$ and

$$
\ell:=\frac{k \chi-\sum_{x \in I} \sum_{i=1}^{l_{x}} d_{i}(x) r_{i}(x)}{r} .
$$

Then $\mathcal{U}_{C, \omega}$ is the GIT quotient $\mathcal{R}_{\omega}^{s s} \stackrel{\psi}{\rightarrow} \mathcal{U}_{C, \omega}:=\mathcal{U}_{C}(r, d, \omega)$ and $\Theta_{\mathcal{R}^{s s}, \omega}$ descends to an ample line bundle $\Theta_{\mathcal{U}_{C, \omega}}$ on $\mathcal{U}_{C, \omega}$ when $\ell$ is an integer.

Definition 3.4. When $C$ is a smooth projective curve, let

$$
\text { Det }: \mathcal{U}_{C, \omega} \rightarrow J_{C}^{d}, \quad E \mapsto \operatorname{det}(E):=\bigwedge^{r} E
$$

be the determinant map. Then, for any $L \in J_{C}^{d}$, the fiber

$$
\operatorname{Det}^{-1}(L):=\mathcal{U}_{C, \omega}^{L}
$$

is called moduli space of semistable parabolic bundles with a fixed determinant. 
Let $\mathcal{R}_{F}^{L} \subset \mathcal{R}$ be the sub-scheme of locally free sheaves with a fixed determinant $L$, and $\left(\mathcal{R}_{\omega}^{s s}\right)^{L} \subset \mathcal{R}_{\omega}^{s s},\left(\mathcal{R}_{\omega}^{s}\right)^{L} \subset \mathcal{R}_{\omega}^{s}$ be the closed subsets of locally free sheaves with the fixed determinant $L$. Then $\mathcal{U}_{C, \omega}^{L}$ is the GIT quotient $\left(\mathcal{R}_{\omega}^{s s}\right)^{L} \stackrel{\psi}{\rightarrow}\left(\mathcal{R}_{\omega}^{s s}\right)^{L} / / \operatorname{SL}(V):=\mathcal{U}_{C, \omega}^{L}$. The proof of globally F-regular type of $\mathcal{U}_{C, \omega}^{L}$ needs essentially the following two results.

Proposition 3.5. Let $|\mathrm{I}|$ be the number of parabolic points. Then, for any data $\omega=\left(k,\{\vec{n}(x), \vec{a}(x)\}_{x \in I}\right)$, we have

(1) $\operatorname{Codim}\left(\left(\mathcal{R}_{\omega}^{s s}\right)^{L} \backslash\left(\mathcal{R}_{\omega}^{s}\right)^{L}\right) \geq(r-1)(g-1)+\frac{1}{k}|\mathrm{I}|$,

(2) $\operatorname{Codim}\left(\mathcal{R}_{F}^{L} \backslash\left(\mathcal{R}_{\omega}^{s s}\right)^{L}\right)>(r-1)(g-1)+\frac{1}{k}|\mathrm{I}|$.

Proof. This is in fact Proposition 5.1 of [16] where we did not fix determinant and the term $\frac{1}{k}|\mathrm{I}|$ was omitted. However, the proof also works for the case of fixed determinant.

Proposition 3.6. Let $\omega_{c}=\left(2 r,\left\{\vec{n}(x), \vec{a}_{c}(x)\right\}_{x \in I}\right)$, where

$$
\vec{a}_{c}(x)=\left(\bar{a}_{1}(x), \bar{a}_{2}(x), \cdots, \bar{a}_{l_{x}+1}(x)\right)
$$

satisfy $\bar{a}_{i+1}(x)-\bar{a}_{i}(x)=n_{i}(x)+n_{i+1}(x)\left(1 \leq i \leq l_{x}\right)$. Then, when

$$
(r-1)(g-1)+\frac{|I|}{2 r} \geq 2
$$

the moduli space $\mathcal{U}_{C, \omega_{c}}^{L}=\left(\mathcal{R}_{\omega_{c}}^{s s}\right)^{L} / / \mathrm{SL}(V)$ is a normal Fano variety with only rational singularities.

Proof. It is in fact a reformulation of Proposition 2.2 of [16] where a formula of anti-canonical bundle $\omega_{\mathcal{R}_{F}}^{-1}$ (thus a formula of $\omega_{\mathcal{R}_{F}^{L}}^{-1}$ ) was given (see also Proposition 4.2 of [18] for a tidier formula). The line bundle $\omega_{\mathcal{R}_{F}^{L}}^{-1}$ is precisely determined by the data $\omega_{c}$ and descends to an ample line bundle $\Theta_{\mathcal{U}_{C}^{L}, \omega_{c}}$, which is precisely $\omega_{\mathcal{U}_{C, \omega_{C}}^{L}}^{-1}$ when

$$
\operatorname{Codim}\left(\left(\mathcal{R}_{\omega_{c}}^{s s}\right)^{L} \backslash\left(\mathcal{R}_{\omega_{c}}^{s}\right)^{L}\right) \geq 2
$$

by a result of F. Knop (see [3]). Thus we are done by the condition (3.1) and (1) of Proposition 3.5.

Theorem 3.7. The moduli spaces $\mathcal{U}_{C, \omega}^{L}$ are of globally F-regular type. If Jacobian $J_{C}^{0}$ of $C$ is of F-split type, so is $\mathcal{U}_{C, \omega}$.

Proof. Choose a subset $I^{\prime} \subset C$ such that $I^{\prime} \cap I=\emptyset$ and

$$
(r-1)(g-1)+\frac{|I|+\left|I^{\prime}\right|}{2 r} \geq 2 .
$$

Let

$$
\mathcal{R}^{\prime}=\underset{x \in I \cup I^{\prime}}{\times_{\mathbf{Q}}} \operatorname{Flag} \operatorname{lag}_{\vec{n}(x)}\left(\mathcal{F}_{x}\right)=\mathcal{R} \times_{\mathbf{Q}}\left(\underset{x \in I^{\prime}}{\times_{\mathbf{Q}}} \operatorname{Flag}_{\vec{n}(x)}\left(\mathcal{F}_{x}\right)\right) \stackrel{\hat{f}}{\rightarrow} \mathcal{R}
$$


be the projection, $\hat{Y} \subset \mathcal{R}$ be the Zariski closure of $\mathcal{R}_{F}^{L}$ and

$$
\hat{Z}=\hat{f}^{-1}(\hat{Y}) \subset \mathcal{R}^{\prime}, \quad \mathcal{R}_{F}^{L}=\hat{f}^{-1}\left(\mathcal{R}_{F}^{L}\right) \subset \hat{Z} .
$$

Then $\mathcal{R}_{F}^{\prime L} \subset \hat{Z}, \mathcal{R}_{F}^{L} \subset \hat{Y}$ are normal (in fact, smooth) $\mathrm{SL}(V)$-invariant open sub-schemes such that $\hat{Y}_{\omega}^{s s}=\left(\mathcal{R}_{\omega}^{s s}\right)^{L} \subset \mathcal{R}_{F}^{L}, \hat{Z}_{\omega^{\prime}}^{s s}=\left(\mathcal{R}_{\omega^{\prime}}^{\prime s s}\right)^{L} \subset \mathcal{R}_{F}^{\prime L}$ holds for any polarizations determined by data $\omega, \omega^{\prime}$. It is clear that $\mathcal{R}_{F}^{\prime L} \stackrel{\hat{f}}{\rightarrow} \mathcal{R}_{F}^{L}$ is a flag bundle and $p$-compatible with $\hat{f}_{*} \mathcal{O}_{\mathcal{R}_{F}^{\prime L}}=\mathcal{O}_{\mathcal{R}_{F}^{L}}$. Thus $\mathcal{R}_{F}^{\prime L} \subset \hat{Z}, \mathcal{R}_{F}^{L} \subset \hat{Y}, \mathcal{R}_{F}^{\prime L} \stackrel{\hat{f}}{\rightarrow} \mathcal{R}_{F}^{L}$ satisfy the conditions (1) and (2) of Proposition 2.10, To verify condition (3) in Proposition 2.10, let

$$
W:=\hat{Z}_{\omega^{\prime}}^{s}=\left(\mathcal{R}_{\omega^{\prime}}^{\prime s}\right)^{L} \subset \mathcal{R}_{F}^{\prime L}, \quad \hat{X}=\hat{f}^{-1}\left(\left(\mathcal{R}_{\omega}^{s s}\right)^{L}\right) \cap W,
$$

$\hat{Z}_{\omega^{\prime}}^{s s} \stackrel{\varphi}{\rightarrow} Z:=\hat{Z}_{\omega^{\prime}}^{s s} / / \operatorname{SL}(V)$ and $X=\varphi(\hat{X}) \subset Z$. It is clear that

$$
\hat{X}=\varphi^{-1}(X) \text {. }
$$

If we choose $\omega^{\prime}=\left(2 r,\left\{\vec{n}(x), \vec{a}_{c}(x)\right\}_{x \in I \cup I^{\prime}}\right)$ in Proposition 3.6, then

$$
\operatorname{Codim}\left(\mathcal{R}_{F}^{\prime L} \backslash W\right) \geq(r-1)(g-1)+\frac{|I|+\left|I^{\prime}\right|}{2 r} \geq 2
$$

by Proposition 3.5 , and $Z$ is a normal Fano variety with only rational singularities. Thus $Z$ is of globally F-regular type by Proposition 2.6, so is $\mathcal{U}_{C, \omega}^{L}=\left(\mathcal{R}_{\omega}^{s s}\right)^{L} / / \mathrm{SL}(V)=\hat{Y}_{\omega}^{s s} / / \mathrm{SL}(V)$ by Proposition 2.10.

If $J_{C}^{0}$ is of F-split type, so is $J_{C}^{0} \times \mathcal{U}_{C, \omega}^{L}$. We have a $r^{2 g}$-fold covering

$$
J_{C}^{0} \times \mathcal{U}_{C, \omega}^{L} \stackrel{f}{\rightarrow} \mathcal{U}_{C, \omega}, \quad f\left(\mathcal{L}_{0}, E\right)=\mathcal{L}_{0} \otimes E
$$

which implies that $\mathcal{U}_{C, \omega}$ is of F-split type.

\section{Globally F-Regular type of Moduli spaces of GENERALIZED PARABOLIC SHEAVES}

In this section, we prove that moduli spaces of generalized parabolic sheaves with a fixed determinant on a smooth curve are of globally F-regular type. We will continue to use notations of last section.

Let $\left\{x_{1}, x_{2}\right\} \subset C \backslash I$ be two different points, a generalized parabolic sheaf (GPS) $(E, Q)$ of rank $r$ and degree $d$ on $C$ consists of a sheaf $E$ of degree $d$ on $C$, torsion free of rank $r$ outside $\left\{x_{1}, x_{2}\right\}$ with parabolic structures at the points of $I$ and an $r$-dimensional quotient

$$
E_{x_{1}} \oplus E_{x_{2}} \stackrel{q}{\rightarrow} Q \rightarrow 0
$$


Definition 4.1. A GPS $(E, Q)$ on an irreducible smooth curve $C$ is called semistable (resp., stable), if for every nontrivial subsheaf $E^{\prime} \subset E$ such that $E / E^{\prime}$ is torsion free outside $\left\{x_{1}, x_{2}\right\}$, we have

$$
\operatorname{par} \chi\left(E^{\prime}\right)-\operatorname{dim}\left(Q^{E^{\prime}}\right) \leq r\left(E^{\prime}\right) \cdot \frac{\operatorname{par} \chi(E)-\operatorname{dim}(Q)}{r(E)} \quad \text { (resp., <), }
$$

where $Q^{E^{\prime}}=q\left(E_{x_{1}}^{\prime} \oplus E_{x_{2}}^{\prime}\right) \subset Q$.

Theorem 4.2 (Theorem X2 of [10] or Theorem 2.24 of [18] for arbitrary rank). For any data $\omega=\left(k,\{\vec{n}(x), \vec{a}(x)\}_{x \in I}\right)$, there exists a normal projective variety $\mathcal{P}_{\omega}$ with at most rational singularities, which is the coarse moduli space of s-equivalence classes of semi-stable GPS on $C$ with parabolic structures at the points of I given by the data $\omega$.

Recall the construction of $\mathcal{P}_{\omega}$. Let $\operatorname{Grass}_{r}\left(\mathcal{F}_{x_{1}} \oplus \mathcal{F}_{x_{2}}\right) \rightarrow \mathbf{Q}$ and

$$
\widetilde{\mathcal{R}}=\operatorname{Grass}_{r}\left(\mathcal{F}_{x_{1}} \oplus \mathcal{F}_{x_{2}}\right) \times_{\mathbf{Q}} \mathcal{R} \stackrel{\rho}{\rightarrow} \mathcal{R} .
$$

$\omega=\left(k,\{\vec{n}(x), \vec{a}(x)\}_{x \in I}\right)$ determines a polarization, which linearizes the $\mathrm{SL}(V)$-action on $\widetilde{\mathcal{R}}$, such that the open set $\widetilde{\mathcal{R}}_{\omega}^{s s}$ (resp. $\widetilde{\mathcal{R}}_{\omega}^{s}$ ) of GIT semistable (resp. GIT stable) points are precisely the set of semistable (resp. stable) GPS on $C$ (see [18]). Then $\mathcal{P}_{\omega}$ is the GIT quotient

$$
\widetilde{\mathcal{R}}_{\omega}^{s s} \stackrel{\psi}{\rightarrow} \widetilde{\mathcal{R}}_{\omega}^{s s} / / \mathrm{SL}(V):=\mathcal{P}_{\omega} .
$$

Notation 4.3. Let $\mathcal{H} \subset \widetilde{\mathcal{R}}$ be the open subscheme parametrising the generalised parabolic sheaves $E=\left(E, E_{x_{1}} \oplus E_{x_{2}} \stackrel{q}{\rightarrow} Q\right)$ satisfying

(1) the torsion Tor $E$ of $E$ is supported on $\left\{x_{1}, x_{2}\right\}$ and

$$
q:(\text { Tor } E)_{x_{1}} \oplus(\text { Tor } E)_{x_{2}} \hookrightarrow Q
$$

(2) if $N$ is large enough, then $H^{1}\left(E(N)\left(-x-x_{1}-x_{2}\right)\right)=0$ for all $E$ and $x \in C$.

Then $\mathcal{H}$ is reduced, normal, Gorenstein with at most rational singularities (see Proposition 3.2 and Remark 3.1 of [16]). Moreover, for any data $\omega$, we have $\widetilde{\mathcal{R}}_{\omega}^{s s} \subset \mathcal{H}$ and, by Lemma 5.7 of [16], there is a

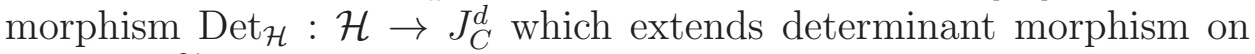
open set $\widetilde{\mathcal{R}}_{F} \subset \mathcal{H}$ of locally free sheaves, and induces a flat morphism

$$
\text { Det }: \mathcal{P}_{\omega} \rightarrow J_{C}^{d} \text {. }
$$

Notation 4.4. For $L \in J_{C}^{d}$, let $\mathcal{H}^{L}=\operatorname{Det}^{-1}(L) \subset \mathcal{H}$,

$$
\widetilde{\mathcal{R}}_{F}^{L}=\operatorname{Det}^{-1}(L) \subset \widetilde{\mathcal{R}}_{F}, \quad\left(\widetilde{\mathcal{R}}_{\omega}^{s s}\right)^{L}=\operatorname{Det}^{-1}(L) \subset \widetilde{\mathcal{R}}_{\omega}^{s s} .
$$

Then $\mathcal{P}_{\omega}^{L}=\operatorname{Det}^{-1}(L) \subset \mathcal{P}_{\omega}$ is the GIT quotient

$$
\left(\widetilde{\mathcal{R}}_{\omega}^{s s}\right)^{L} \stackrel{\psi}{\rightarrow} \mathcal{P}_{\omega}^{L}=\left(\widetilde{\mathcal{R}}_{\omega}^{s s}\right)^{L} / / \mathrm{SL}(V) .
$$


Proposition 4.5 (Proposition 5.2 of [16]). Let $\mathcal{D}_{1}^{f}=\hat{\mathcal{D}}_{1} \cup \hat{\mathcal{D}}_{1}^{t}$ and $\mathcal{D}_{2}^{f}=\hat{\mathcal{D}}_{2} \cup \hat{\mathcal{D}}_{2}^{t}$, where $\hat{\mathcal{D}}_{i} \subset \widetilde{\mathcal{R}}$ is the Zariski closure of $\hat{\mathcal{D}}_{F, i} \subset \widetilde{\mathcal{R}}_{F}$ consisting of $(E, Q) \in \widetilde{\mathcal{R}}_{F}$ that $E_{x_{i}} \rightarrow Q$ is not an isomorphism, and $\hat{\mathcal{D}}_{1}^{t} \subset \widetilde{\mathcal{R}}\left(\right.$ rep. $\left.\hat{\mathcal{D}}_{2}^{t} \subset \widetilde{\mathcal{R}}\right)$ consists of $(E, Q) \in \widetilde{\mathcal{R}}$ such that $E$ is not locally free at $x_{2}$ (resp. at $\left.x_{1}\right)$. Then

(1) $\operatorname{Codim}\left(\mathcal{H}^{L} \backslash\left(\widetilde{\mathcal{R}}_{\omega}^{s s}\right)^{L}\right)>(r-1) g+\frac{|I|}{k}$;

(2) the complement in $\left(\widetilde{\mathcal{R}}_{\omega}^{s s}\right)^{L} \backslash\left\{\mathcal{D}_{1}^{f} \cup \mathcal{D}_{2}^{f}\right\}$ of the set $\widetilde{\mathcal{R}}_{\omega}^{s}$ of stable points has codimension $\geq(r-1) g+\frac{|I|}{k}$.

(3) $\operatorname{Codim}\left(\left(\widetilde{\mathcal{R}}_{\omega}^{s s}\right)^{L} \backslash W_{\omega}\right) \geq(r-1) g+\frac{|I|}{k}, W_{\omega} \subset\left(\widetilde{\mathcal{R}}_{\omega}^{s s}\right)^{L}$ defined by

$W_{\omega}:=\left\{(E, Q) \in\left(\widetilde{\mathcal{R}}_{\omega}^{s s}\right)^{L} \mid \begin{array}{l}\forall E^{\prime} \subset E \text { with } 0<r\left(E^{\prime}\right)<r, \text { we have } \\ \frac{\operatorname{par} \chi\left(E^{\prime}\right)-\operatorname{dim}\left(Q^{E^{\prime}}\right)}{r\left(E^{\prime}\right)}<\frac{\operatorname{par} \chi(E)-\operatorname{dim}(Q)}{r(E)}\end{array}\right\}$.

Proof. The statements (1) and (2) are contained in Proposition 5.2 of [16] (where the term $\frac{|I|}{k}$ was omitted). The proof of Proposition 5.2 (2) in [16] implies statement (3) here.

Proposition 4.6. Let $\omega_{c}=\left(2 r,\left\{\vec{n}(x), \vec{a}_{c}(x)\right\}_{x \in I}\right)$ be the data in Proposition 3.6 and $\Theta_{J_{C}^{d}}$ be the theta line bundle on $J_{C}^{d}$. Assume

$$
(r-1)(g-1)+\frac{|I|}{2 r} \geq 2 .
$$

Then there is an ample line bundle $\Theta_{\mathcal{P}_{\omega_{c}}}$ on $\mathcal{P}_{\omega_{c}}$ such that

$$
\omega_{\mathcal{P}_{\omega_{c}}}^{-1}=\Theta_{\mathcal{P}_{\omega_{c}}} \otimes \operatorname{Det}^{*}\left(\Theta_{J_{C}^{d}}^{-1}\right) .
$$

In particular, for any $L \in J_{C}^{d}$, $\mathcal{P}_{\omega_{c}}^{L}$ is a normal Fano variety with only rational singularities.

Proof. Let $V \otimes \mathcal{O}_{C \times \mathcal{H}}(-N) \rightarrow \mathcal{E} \rightarrow 0, \quad \mathcal{E}_{x_{1}} \oplus \mathcal{E}_{x_{2}} \rightarrow \mathcal{Q} \rightarrow 0$ and

$$
\left\{\mathcal{E}_{\{x\} \times \mathcal{H}}=\mathcal{Q}_{\{x\} \times \mathcal{H}, l_{x}+1} \rightarrow \mathcal{Q}_{\{x\} \times \mathcal{H}, l_{x}} \rightarrow \cdots \rightarrow \mathcal{Q}_{\{x\} \times \mathcal{H}, 1} \rightarrow 0\right\}_{x \in I}
$$

be the universal quotients and universal flags. Let $\omega_{C}=\mathcal{O}\left(\sum_{q} q\right)$ and

$$
\Theta_{J_{C}^{d}}=\left(\operatorname{det} R \pi_{J_{C}^{d}} \mathcal{L}\right)^{-2} \otimes \mathcal{L}_{x_{1}}^{r} \otimes \mathcal{L}_{x_{2}}^{r} \otimes \mathcal{L}_{y}^{2 \chi-2 r} \otimes \bigotimes_{q} \mathcal{L}_{q}^{r-1}
$$


where $\mathcal{L}$ is the universal line bundle on $C \times J_{C}^{d}$. Then we have

$$
\begin{aligned}
& \omega_{\mathcal{H}}^{-1}=\left(\operatorname{det} R \pi_{\mathcal{H}} \mathcal{E}\right)^{-2 r} \otimes \\
& \bigotimes_{x \in I}\left\{\left(\operatorname{det} \mathcal{E}_{x}\right)^{n_{l_{x}+1}-r} \otimes \bigotimes_{i=1}^{l_{x}}\left(\operatorname{det} \mathcal{Q}_{x, i}\right)^{n_{i}(x)+n_{i+1}(x)}\right\} \otimes(\operatorname{det} \mathcal{Q})^{2 r} \\
& \otimes\left(\operatorname{det} \mathcal{E}_{y}\right)^{2 \chi-2 r} \otimes \operatorname{Det}_{\mathcal{H}}^{*}\left(\Theta_{J_{C}^{d}}^{-1}\right):=\hat{\Theta}_{\omega_{c}} \otimes \operatorname{Det}_{\mathcal{H}}^{*}\left(\Theta_{J_{C}^{d}}^{-1}\right)
\end{aligned}
$$

by Proposition 3.4 of [16], and $\hat{\Theta}_{\omega_{c}}$ descends to an ample line bundle $\Theta_{\mathcal{P}_{\omega_{c}}}$ on $\mathcal{P}_{\omega_{c}}$ (see Lemma 2.3 of [16]). Thus

$$
\left(\psi_{*} \omega_{\widetilde{\mathcal{R}}_{\omega_{c}}^{s s}}^{-1}\right)^{i n v .}=\Theta_{\mathcal{P}_{\omega_{c}}} \otimes \operatorname{Det}^{*}\left(\Theta_{J_{C}^{d}}^{-1}\right) \text {. }
$$

When condition (4.3) holds, the lower bounds in Proposition 3.5 and Proposition 4.5 are at least two. Thus Lemma 5.6 of [16] is applicable (where assumption $g \geq 2$ in Lemma 5.6 of [16] is replaced by condition (4.3) ) and $\left(\psi_{*} \omega_{\widetilde{\mathcal{R}}_{\omega_{c}}^{s s}}^{-1}\right)^{i n v .}=\omega_{\mathcal{P}_{\omega_{c}}}^{-1}$.

Theorem 4.7. For any data $\omega=\left(k,\{\vec{n}(x), \vec{a}(x)\}_{x \in I}\right)$, the moduli space $\mathcal{P}_{\omega}^{L}$ is of globally F-regular type.

Proof. Choose a finite subset $I^{\prime} \subset C \backslash I$ satisfying (4.3). Recall that

$$
\mathcal{R}=\underset{x \in I}{\times_{\mathbf{Q}}} \operatorname{Flag}_{\vec{n}(x)}\left(\mathcal{F}_{x}\right), \quad \mathcal{R}^{\prime}=\underset{x \in I \cup I^{\prime}}{\times_{\mathbf{Q}}} \operatorname{Flag} \operatorname{lag}_{\vec{n}(x)}\left(\mathcal{F}_{x}\right) \stackrel{\hat{f}}{\rightarrow} \mathcal{R}
$$

be the projection and $\widetilde{\mathcal{R}}=\operatorname{Grass}_{r}\left(\mathcal{F}_{x_{1}} \oplus \mathcal{F}_{x_{2}}\right) \times_{\mathbf{Q}} \mathcal{R} \stackrel{\rho}{\rightarrow} \mathcal{R}$. Let

$$
\widetilde{\mathcal{R}}^{\prime}:=\operatorname{Grass}_{r}\left(\mathcal{F}_{x_{1}} \oplus \mathcal{F}_{x_{2}}\right) \times_{\mathbf{Q}} \mathcal{R}^{\prime} \stackrel{\hat{f}}{\rightarrow} \widetilde{\mathcal{R}} .
$$

Then, on $\mathcal{H}^{L} \subset \widetilde{\mathcal{R}}$, it is clear that $\left(\mathcal{H}^{\prime}\right)^{L}:=\hat{f}^{-1}\left(\mathcal{H}^{L}\right) \stackrel{\hat{f}}{\rightarrow} \mathcal{H}^{L}$ is a $\operatorname{SL}(V)$ invariant and $p$-compatible morphism such that $\hat{f}_{*} \mathcal{O}_{\left(\mathcal{H}^{\prime}\right)^{L}}=\mathcal{O}_{\mathcal{H}^{L}}$.

For any data $\omega=\left(k,\{\vec{n}(x), \vec{a}(x)\}_{x \in I}\right), \omega_{c}=\left(2 r,\left\{\vec{n}(x), \vec{a}_{c}(x)\right\}_{x \in I \cup I^{\prime}}\right)$, we have $\left(\widetilde{\mathcal{R}}_{\omega}^{s s}\right)^{L} \subset \mathcal{H}^{L},\left(\widetilde{\mathcal{R}}_{\omega_{c}}^{\prime s s}\right)^{L} \subset\left(\mathcal{H}^{\prime}\right)^{L}$. Recall

$$
\left(\widetilde{\mathcal{R}}_{\omega}^{s s}\right)^{L} \stackrel{\psi}{\rightarrow} \mathcal{P}_{\omega}^{L}:=Y, \quad\left(\widetilde{\mathcal{R}}_{\omega_{c}}^{\prime s s}\right)^{L} \stackrel{\varphi}{\rightarrow} \mathcal{P}_{\omega_{c}}^{L}:=Z .
$$

To apply Proposition 2.10, let $W=W_{\omega_{c}} \subset\left(\widetilde{\mathcal{R}}_{\omega_{c}}^{s s}\right)^{L}$ and

$$
\hat{X}=W \cap \hat{f}^{-1}\left(\left(\widetilde{\mathcal{R}}_{\omega}^{s s}\right)^{L}\right) .
$$

By Proposition 4.5, $\operatorname{Codim}\left(\left(\mathcal{H}^{\prime}\right)^{L} \backslash W\right) \geq(r-1) g+\frac{|I|+\left|I^{\prime}\right|}{2 r} \geq 2$. Thus it is enough to check the condition that $\hat{X}=\varphi^{-1} \varphi(\hat{X})$. This is equivalent (see Remark 1.2 of [16]) to prove that

$$
\forall(E, Q) \in\left(\widetilde{\mathcal{R}}_{\omega_{c}}^{\prime s s}\right)^{L}, \quad(E, Q) \in \hat{X} \quad \Leftrightarrow \quad \operatorname{gr}(E, Q) \in \hat{X} .
$$


In fact, for any $(E, Q) \in\left(\widetilde{\mathcal{R}}_{\omega_{c}}^{s s}\right)^{L}$, it is clear that we have

$$
(E, Q) \in W \Leftrightarrow \operatorname{gr}(E, Q)=(\widetilde{E}, \widetilde{Q}) \oplus\left({ }_{x_{1}} \tau_{1} \oplus{ }_{x_{2}} \tau_{2}, \tau_{1} \oplus \tau_{2}\right) \in W
$$

where $(\widetilde{E}, \widetilde{Q})$ is a stable GPS (see Definition 1.5 of [16]). Thus either

$$
0 \rightarrow\left({ }_{x_{1}} \tau_{1} \oplus{ }_{x_{2}} \tau_{2}, \tau_{1} \oplus \tau_{2}\right) \rightarrow(E, Q) \rightarrow(\widetilde{E}, \widetilde{Q}) \rightarrow 0
$$

or $0 \rightarrow\left(E^{\prime}, Q^{\prime}\right) \rightarrow(E, Q) \rightarrow\left({ }_{x_{i}} \mathbb{C}, \mathbb{C}\right) \rightarrow 0$. Then $(E, Q)$ is semi-stable (respect to $\omega$ ) if and only if $(\widetilde{E}, \widetilde{Q})$ is semi-stable (respect to $\omega$ ). Thus (4.5) is proved and we are done.

When $C=C_{1} \cup C_{2}$ is reducible with two smooth irreducible components $C_{1}$ and $C_{2}$ of genus $g_{1}$ and $g_{2}$ meeting at only one point $x_{0}$ (which is the only node of $C$ ), we fix an ample line bundle $\mathcal{O}(1)$ of degree $c$ on $C$ such that $\operatorname{deg}\left(\left.\mathcal{O}(1)\right|_{C_{i}}\right)=c_{i}>0(i=1,2)$. For any coherent sheaf $E$, $P(E, n):=\chi(E(n))$ denotes its Hilbert polynomial, which has degree 1. We define the rank of $E$ to be

$$
r(E):=\frac{1}{\operatorname{deg}(\mathcal{O}(1))} \cdot \lim _{n \rightarrow \infty} \frac{P(E, n)}{n} .
$$

Let $r_{i}$ denote the rank of the restriction of $E$ to $C_{i}(i=1,2)$, then

$$
P(E, n)=\left(c_{1} r_{1}+c_{2} r_{2}\right) n+\chi(E), \quad r(E)=\frac{c_{1}}{c_{1}+c_{2}} r_{1}+\frac{c_{2}}{c_{1}+c_{2}} r_{2} .
$$

We say that $E$ is of rank $r$ on $X$ if $r_{1}=r_{2}=r$, otherwise it will be said of rank $\left(r_{1}, r_{2}\right)$.

Fix a finite set $I=I_{1} \cup I_{2}$ of smooth points on $C$, where $I_{i}=\{x \in$ $\left.I \mid x \in C_{i}\right\}(i=1,2)$, and parabolic data $\omega=\{k, \vec{n}(x), \vec{a}(x)\}_{x \in I}$ with

$$
\ell:=\frac{k \chi-\sum_{x \in I} \sum_{i=1}^{l_{x}} d_{i}(x) r_{i}(x)}{r}
$$

$\left(\right.$ recall $\left.d_{i}(x)=a_{i+1}(x)-a_{i}(x), r_{i}(x)=n_{1}(x)+\cdots+n_{i}(x)\right)$. Let

$$
n_{j}^{\omega}=\frac{1}{k}\left(r \frac{c_{j}}{c_{1}+c_{2}} \ell+\sum_{x \in I_{j}} \sum_{i=1}^{l_{x}} d_{i}(x) r_{i}(x)\right) \quad(j=1,2) .
$$

Definition 4.8. For any coherent sheaf $F$ of rank $\left(r_{1}, r_{2}\right)$, let

$$
m(F):=\frac{r(F)-r_{1}}{k} \sum_{x \in I_{1}} a_{l_{x}+1}(x)+\frac{r(F)-r_{2}}{k} \sum_{x \in I_{2}} a_{l_{x}+1}(x),
$$

the modified parabolic Euler characteristic and slop of $F$ are

$$
\operatorname{par} \chi_{m}(F):=\operatorname{par} \chi(F)+m(F), \quad \operatorname{par} \mu_{m}(F):=\frac{\operatorname{par} \chi_{m}(F)}{r(F)} .
$$


A parabolic sheaf $E$ is called semistable (resp. stable) if, for any subsheaf $F \subset E$ such $E / F$ is torsion free, one has, with the induced parabolic structure,

$$
\operatorname{par} \chi_{m}(F) \leq \frac{\operatorname{par} \chi_{m}(E)}{r(E)} r(F) \quad(\text { resp. }<) .
$$

Theorem 4.9 (Theorem 1.1 of [17] or Theorem 2.14 of [18]). There exists a reduced, seminormal projective scheme

$$
\mathcal{U}_{C}:=\mathcal{U}_{C}\left(r, d, \mathcal{O}(1),\{k, \vec{n}(x), \vec{a}(x)\}_{x \in I_{1} \cup I_{2}}\right)
$$

which is the coarse moduli space of s-equivalence classes of semistable parabolic sheaves $E$ of rank $r$ and $\chi(E)=\chi=d+r(1-g)$ with parabolic structures of type $\{\vec{n}(x)\}_{x \in I}$ and weights $\{\vec{a}(x)\}_{x \in I}$ at points $\{x\}_{x \in I}$. The moduli space $\mathcal{U}_{C}$ has at most $r+1$ irreducible components.

The normalization of $\mathcal{U}_{C}$ is a moduli space of semistable GPS on $\widetilde{C}=C_{1} \bigsqcup C_{2}$ with parabolic structures at points $x \in I$. Recall

Definition 4.10. A $\operatorname{GPS}\left(E, E_{x_{1}} \oplus E_{x_{2}} \stackrel{q}{\rightarrow} Q\right)$ is called semistable (resp., stable), if for every nontrivial subsheaf $E^{\prime} \subset E$ such that $E / E^{\prime}$ is torsion free outside $\left\{x_{1}, x_{2}\right\}$, we have, with the induced parabolic structures at points $\{x\}_{x \in I}$,

$$
\operatorname{par}_{m}\left(E^{\prime}\right)-\operatorname{dim}\left(Q^{E^{\prime}}\right) \leq r\left(E^{\prime}\right) \cdot \frac{\operatorname{par} \chi_{m}(E)-\operatorname{dim}(Q)}{r(E)} \quad(\text { resp. },<),
$$

where $Q^{E^{\prime}}=q\left(E_{x_{1}}^{\prime} \oplus E_{x_{2}}^{\prime}\right) \subset Q$.

Theorem 4.11 (Theorem 2.1 of [17] or Theorem 2.26 of [18]). For any data $\omega=\left(\{k, \vec{n}(x), \vec{a}(x)\}_{x \in I_{1} \cup I_{2}}, \mathcal{O}(1)\right)$, the coarse moduli space $\mathcal{P}_{\omega}$ of s-equivalence classes of semi-stable GPS on $\widetilde{C}$ with parabolic structures at the points of $I$ given by the data $\omega$ is a disjoint union of at most $r+1$ irreducible, normal projective varieties $\mathcal{P}_{\chi_{1}, \chi_{2}}\left(\chi_{1}+\chi_{2}=\chi+r\right.$, $\left.n_{j}^{\omega} \leq \chi_{j} \leq n_{j}^{\omega}+r\right)$ with at most rational singularities.

For fixed $\chi_{1}, \chi_{2}$ satisfying $\chi_{1}+\chi_{2}=\chi+r$ and $n_{j}^{\omega} \leq \chi_{j} \leq n_{j}^{\omega}+r$ $(j=1,2)$, recall the construction of $\mathcal{P}_{\omega}=\mathcal{P}_{\chi_{1}, \chi_{2}}$. Let

$$
P_{i}(m)=c_{i} r m+\chi_{i}, \quad \mathcal{W}_{i}=\mathcal{O}_{C_{i}}(-N), \quad V_{i}=\mathbb{C}^{P_{i}(N)}
$$

where $\mathcal{O}_{C_{i}}(1)=\left.\mathcal{O}(1)\right|_{C_{i}}$ has degree $c_{i}$. Consider the Quot schemes $\mathbf{Q}_{i}=\operatorname{Quot}\left(V_{i} \otimes \mathcal{W}_{i}, P_{i}\right)$, the universal quotient $V_{i} \otimes \mathcal{W}_{i} \rightarrow \mathcal{F}^{i} \rightarrow 0$ on $C_{i} \times \mathbf{Q}_{i}$ and the relative flag scheme

$$
\mathcal{R}_{i}=\underset{x \in I_{i}}{\times \mathbf{Q}_{i}} \operatorname{Flag}_{\vec{n}(x)}\left(\mathcal{F}_{x}^{i}\right) \rightarrow \mathbf{Q}_{i} .
$$


Let $\mathcal{F}=\mathcal{F}^{1} \oplus \mathcal{F}^{2}$ denote direct sum of pullbacks of $\mathcal{F}^{1}, \mathcal{F}^{2}$ on

$$
\widetilde{C} \times\left(\mathbf{Q}_{1} \times \mathbf{Q}_{2}\right)=\left(C_{1} \times \mathbf{Q}_{1}\right) \sqcup\left(C_{2} \times \mathbf{Q}_{2}\right) .
$$

Let $\mathcal{E}$ be the pullback of $\mathcal{F}$ to $\widetilde{C} \times\left(\mathcal{R}_{1} \times \mathcal{R}_{2}\right)$, and

$$
\rho: \widetilde{\mathcal{R}}=\operatorname{Grass}_{r}\left(\mathcal{E}_{x_{1}} \oplus \mathcal{E}_{x_{2}}\right) \rightarrow \mathcal{R}=\mathcal{R}_{1} \times \mathcal{R}_{2} \rightarrow \mathrm{Q}=\mathbf{Q}_{1} \times \mathbf{Q}_{2} .
$$

For the given $\omega=\left(\{k, \vec{n}(x), \vec{a}(x)\}_{x \in I_{1} \cup I_{2}}, \mathcal{O}(1)\right)$, let $\widetilde{\mathcal{R}}_{\omega}^{s s}\left(\operatorname{resp} . \widetilde{\mathcal{R}}_{\omega}^{s}\right)$ denote the open set of GIT semi-stable (resp. GIT stable) points under action of $G=\left(\operatorname{GL}\left(V_{1}\right) \times \mathrm{GL}\left(V_{2}\right)\right) \cap S L\left(V_{1} \oplus V_{2}\right)$ on $\widetilde{\mathcal{R}}$ respect to the polarization determined by $\omega$. Let $\mathcal{H} \subset \widetilde{\mathcal{R}}$ be the open set defined in Notation 4.3, then for any data $\omega$ we have

$$
\widetilde{\mathcal{R}}_{\omega}^{s} \subset \widetilde{\mathcal{R}}_{\omega}^{s s} \subset \mathcal{H} .
$$

The moduli space in Theorem 4.11 is nothing but the GIT quotient

$$
\psi: \widetilde{\mathcal{R}}_{\omega}^{s s} \rightarrow \mathcal{P}_{\omega}:=\widetilde{\mathcal{R}}_{\omega}^{s s} / / G
$$

There exists a morphism $\hat{\operatorname{Det}}_{\mathcal{H}}: \mathcal{H} \rightarrow J_{\widetilde{C}}^{d}=J_{C_{1}}^{d_{1}} \times J_{C_{2}}^{d_{2}}$, which extends

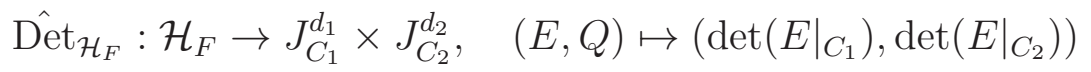

on the open set $\mathcal{H}_{F} \subset \mathcal{H}$ of $\operatorname{GPB}$ (i.e. $\operatorname{GPS}(E, Q)$ with $E$ locally free) and induces a flat determinant morphism

$$
\operatorname{Det}_{\mathcal{P}_{\omega}}: \mathcal{P}_{\omega} \rightarrow J_{\widetilde{C}}^{d}=J_{C_{1}}^{d_{1}} \times J_{C_{2}}^{d_{2}}
$$

(see page 46 of [18] for detail). In fact, for any $L \in J_{\widetilde{C}}^{d}=J_{C_{1}}^{d_{1}} \times J_{C_{2}}^{d_{2}}$, let

$$
\mathcal{P}_{\omega}^{L}:=\operatorname{Det}_{\mathcal{P}_{\omega}}^{-1}(L) \subset \mathcal{P}_{\omega}
$$

and note that abelian variety $J_{C}^{0}=J_{C_{1}}^{0} \times J_{C_{2}}^{0}$ acts on $\mathcal{P}_{\omega}$, the induced morphism $\mathcal{P}_{\omega}^{L} \times J_{X}^{0} \rightarrow \mathcal{P}_{\omega}$ is a finite cover (see the proof of Lemma 6.6 in [18]). Similarly, let $\mathcal{H}^{L}=\hat{\operatorname{Det}}_{\mathcal{H}}^{-1}(L)$ and $\left(\widetilde{\mathcal{R}}_{\omega}^{s s}\right)^{L}=\widetilde{\mathcal{R}}_{\omega}^{s s} \cap \mathcal{H}^{L}$, then

$$
\psi:\left(\widetilde{\mathcal{R}}_{\omega}^{s s}\right)^{L} \rightarrow \mathcal{P}_{\omega}^{L}=\left(\widetilde{\mathcal{R}}_{\omega}^{s s}\right)^{L} / / G .
$$

We do not have good estimate of $\operatorname{Codim}\left(\mathcal{H} \backslash \widetilde{\mathcal{R}}_{\omega}^{s s}\right)$ since sub-sheaves $\left(E_{1}, \operatorname{Tor}\left(E_{2}\right)\right),\left(\operatorname{Tor}\left(E_{1}\right), E_{2}\right)$ of $E=\left(E_{1}, E_{2}\right)$ with rank $(r, 0),(0, r)$ may destroy semi-stability of $(E, Q)$ where $\operatorname{Tor}\left(E_{i}\right) \subset E_{i}(i=1,2)$ are torsion sub-sheaves. But we have estimate of $\operatorname{Codim}\left(\mathcal{H}_{\omega} \backslash \widetilde{\mathcal{R}}_{\omega}^{s s}\right)$, where $\mathcal{H}_{\omega}=\left\{\begin{array}{l}(E, Q) \in \mathcal{H}, \text { with } n_{j}^{\omega} \leq \chi\left(E_{j}\right)=\chi_{j} \leq n_{j}^{\omega}+r(j=1,2), \text { and } \\ \operatorname{dim}\left(\operatorname{Tor}\left(E_{1}\right)\right) \leq n_{2}^{\omega}+r-\chi_{2}, \operatorname{dim}\left(\operatorname{Tor}\left(E_{2}\right)\right) \leq n_{1}^{\omega}+r-\chi_{1}\end{array}\right\}$. 
Proposition 4.12. Let $\mathcal{D}_{1}^{f}=\hat{\mathcal{D}}_{1} \cup \hat{\mathcal{D}}_{1}^{t}$ and $\mathcal{D}_{2}^{f}=\hat{\mathcal{D}}_{2} \cup \hat{\mathcal{D}}_{2}^{t}$, where $\hat{\mathcal{D}}_{i} \subset \widetilde{\mathcal{R}}$ is the Zariski closure of $\hat{\mathcal{D}}_{F, i} \subset \widetilde{\mathcal{R}}_{F}$ consisting of $(E, Q) \in \widetilde{\mathcal{R}}_{F}$ that $E_{x_{i}} \rightarrow Q$ is not an isomorphism, and $\hat{\mathcal{D}}_{1}^{t} \subset \widetilde{\mathcal{R}}\left(\right.$ rep. $\left.\hat{\mathcal{D}}_{2}^{t} \subset \widetilde{\mathcal{R}}\right)$ consists of $(E, Q) \in \widetilde{\mathcal{R}}$ such that $E$ is not locally free at $x_{2}$ (resp. at $\left.x_{1}\right)$. Then

(1) $\operatorname{Codim}\left(\mathcal{H}_{\omega}^{L} \backslash\left(\widetilde{\mathcal{R}}_{\omega}^{s s}\right)^{L}\right)>\min _{1 \leq i \leq 2}\left\{(r-1)\left(g_{i}-\frac{r+3}{4}\right)+\frac{\left|I_{i}\right|}{k}\right\}$;

(2) $\operatorname{Codim}\left(\left(\widetilde{\mathcal{R}}_{\omega}^{s s}\right)^{L} \backslash\left\{\mathcal{D}_{1}^{f} \cup \mathcal{D}_{2}^{f}\right\} \backslash\left(\widetilde{\mathcal{R}}_{\omega}^{s}\right)^{L}\right)>\min _{1 \leq i \leq 2}\left\{(r-1)\left(g_{i}-1\right)+\frac{\left|I_{i}\right|}{k}\right\}$ when $n_{1}^{\omega}<\chi_{1}<n_{1}^{\omega}+r$

(3) $\operatorname{Codim}\left(\left(\widetilde{\mathcal{R}}_{\omega}^{s s}\right)^{L} \backslash\left\{\mathcal{D}_{1}^{f} \cup \mathcal{D}_{2}^{f}\right\} \backslash W_{\omega}\right) \geq \min _{1 \leq i \leq 2}\left\{(r-1)\left(g_{i}-1\right)+\frac{\left|I_{i}\right|}{k}\right\}$ when $\chi_{1}=n_{1}^{\omega}$ or $n_{1}^{\omega}+r$, where

$$
\begin{aligned}
& W_{\omega}:=\left\{(E, Q) \in\left(\widetilde{\mathcal{R}}_{\omega}^{s s}\right)^{L} \mid \begin{array}{l}
\frac{\operatorname{par} \chi\left(E^{\prime}\right)-\operatorname{dim}\left(Q^{E^{\prime}}\right)}{r\left(E^{\prime}\right)}<\frac{\operatorname{par} \chi(E)-\operatorname{dim}(Q)}{r(E)} \\
\forall E^{\prime} \subset E \text { of } \operatorname{rank}\left(r_{1}, r_{2}\right) \neq(0, r),(r, 0),(0,0)
\end{array}\right\} ; \\
& \text { (4) } \operatorname{Codim}\left(\left(\widetilde{\mathcal{R}}_{\omega}^{s s}\right)^{L} \backslash W_{\omega}\right) \geq \min _{1 \leq i \leq 2}\left\{(r-1)\left(g_{i}-\frac{r+3}{4}\right)+\frac{\left|I_{i}\right|}{k}\right\} .
\end{aligned}
$$

Proof. The statements (1), (2) and (3) are in fact reformulations of Proposition 6.3 in [18] where determinants are not fixed. (4) follows the proof of Proposition 6.3 in [18] (see Remark 6.7 (2) of [18]).

Proposition 4.13. For any $\omega$, let $\widetilde{\mathcal{R}}_{\omega}^{s s} \stackrel{\psi}{\rightarrow} \mathcal{P}_{\omega}:=\widetilde{\mathcal{R}}_{\omega}^{s s} / / G$ and assume

$$
\min _{1 \leq i \leq 2}\left\{(r-1)\left(g_{i}-\frac{r+3}{4}\right)+\frac{\left|I_{i}\right|}{k}\right\} \geq 2 .
$$

Then $\left(\psi_{*} \omega_{\widetilde{\mathcal{R}}_{\omega}^{s s}}\right)^{i n v .}=\omega_{\mathcal{P}_{\omega}}$. For $\omega_{c}=\left(2 r,\left\{\vec{n}(x), \vec{a}_{c}(x)\right\}_{x \in I_{1} \cup I_{2}}\right)$ satisfying

$$
\min _{1 \leq i \leq 2}\left\{(r-1)\left(g_{i}-\frac{r+3}{4}\right)+\frac{\left|I_{i}\right|}{2 r}\right\} \geq 2,
$$

there is an ample line bundle $\Theta_{\mathcal{P}_{\omega_{c}}}$ on $\mathcal{P}_{\omega_{c}}$ such that

$$
\omega_{\mathcal{P}_{\omega_{c}}}^{-1}=\Theta_{\mathcal{P}_{\omega_{c}}} \otimes \operatorname{Det}_{\mathcal{P}_{\omega_{c}}}^{*}\left(\Theta_{J_{\tilde{C}}^{d}}^{-1}\right) .
$$

In particular, for any $L \in J_{\widetilde{C}}^{d}$, $\mathcal{P}_{\omega_{c}}^{L}$ is a normal Fano variety with only rational singularities.

Proof. According to a result of Knop in [3] (see Lemma 4.17 of [10] for its global formulation), to prove $\left(\psi_{*} \omega_{\widetilde{\mathcal{R}}_{\omega}^{s s}}\right)^{i n v .}=\omega_{\mathcal{P}_{\omega}}$, it is enough to show that (1) the subset where the action of $G$ is not free has codimension at least two; (2) for every prime divisor $D$ in $\widetilde{\mathcal{R}}_{\omega}^{s s}, \psi(D)$ has codimension at most 1 . 
To verify condition (1), when $n_{1}^{\omega}<\chi_{1}<n_{1}^{\omega}+r$, we have

$$
\operatorname{Codim}\left(\widetilde{\mathcal{R}}_{\omega}^{s s} \backslash\left\{\mathcal{D}_{1}^{f} \cup \mathcal{D}_{2}^{f}\right\} \backslash \widetilde{\mathcal{R}}_{\omega}^{s}\right)>\min _{1 \leq i \leq 2}\left\{(r-1)\left(g_{i}-1\right)+\frac{\left|I_{i}\right|}{k}\right\} \geq 2 .
$$

Note that $\mathcal{D}_{j}^{f}=\hat{\mathcal{D}}_{j} \cup \hat{\mathcal{D}}_{j}^{t}$ where $\hat{\mathcal{D}}_{j}, \hat{\mathcal{D}}_{j}^{t}$ are irreducible, normal subvarieties (see Proposition C.7 of [10]), and the subsets of $\hat{\mathcal{D}}_{j}$ and $\hat{\mathcal{D}}_{j}^{t}$, where the action of $G$ is free, are open subsets. Thus it is enough to find a $(E, Q) \in \mathcal{D}_{j}^{f}(j=1,2)$ such that its automorphisms are only scales. Let $E_{i}^{\prime}(i=1,2)$ be stable parabolic bundles of rank $r$ and $\chi\left(E_{i}^{\prime}\right)=\chi_{i}^{\prime}$ on $C_{i}$ with parabolic structures determined by $\left(k,\{\vec{n}(x), \vec{a}(x)\}_{x \in I_{i}}\right)$. If we take $\chi_{1}^{\prime}=\chi_{1}-1, \chi_{2}^{\prime}=\chi_{2}$, let $E_{1}=E_{1}^{\prime} \oplus{ }_{x_{1}} \mathbb{C} \cdot \beta, E_{2}=E_{2}^{\prime}$ and $E=\left(E_{1}, E_{2}\right)$, the surjection $E_{x_{1}} \oplus E_{x_{2}} \stackrel{q}{\rightarrow} Q$ is defined by any isomorphism $E_{x_{2}} \stackrel{q_{2}}{\rightarrow} Q$ and a linear map $E_{x_{1}}=\left(E_{1}^{\prime}\right)_{x_{1}} \oplus \mathbb{C} \cdot \beta \stackrel{q_{1}}{\rightarrow} Q$ such that $q_{1}(\beta) \neq 0$ and $\left.q_{1}\right|_{\left(E_{1}^{\prime}\right)_{x_{1}}} \neq 0$. Then $(E, Q) \in \hat{\mathcal{D}}_{2}^{t}$ by definition. To see $\operatorname{Aut}((E, Q))=\mathbb{C}^{*}$, let $0 \rightarrow K \rightarrow E_{x_{1}} \oplus E_{x_{2}} \stackrel{q}{\rightarrow} Q \rightarrow 0$ and $(E, Q) \stackrel{\Phi}{\rightarrow}(E, Q)$ be an isomorphism. Then $E \stackrel{\Phi}{\rightarrow} E$ is an isomorphism of parabolic bundles such that $\Phi_{x_{1}+x_{2}}(K)=K$. Since $E_{1}^{\prime}, E_{2}$ are stable, $\left.\Phi\right|_{E_{1}}=\left(\lambda_{1}^{\prime}, \lambda_{1}\right): E_{1}^{\prime} \oplus{ }_{x_{1}} \mathbb{C} \beta \rightarrow E_{1}^{\prime} \oplus{ }_{x_{1}} \mathbb{C} \beta$ and $\left.\Phi\right|_{E_{2}}=\lambda_{2}: E_{2} \rightarrow E_{2}$ for nonzero constants $\lambda_{1}^{\prime}, \lambda_{1}, \lambda_{2}$. The requirement $\Phi_{x_{1}+x_{2}}(K)=K$ implies that $\lambda_{1}^{\prime}=\lambda_{1}=\lambda_{2}$. In fact, $K=\left\{(\alpha, f(\alpha)) \in E_{x_{1}} \oplus E_{x_{2}} \mid \forall \alpha \in E_{x_{1}}\right\}$ where $f=-q_{2}^{-1} q_{1}: E_{x_{1}} \rightarrow E_{x_{2}}$. For any $\alpha=\alpha^{\prime}+\beta \in E_{x_{1}}$, we have

$$
\Phi_{x_{1}+x_{2}}(\alpha, f(\alpha))=\left(\lambda_{1}^{\prime} \alpha^{\prime}+\lambda_{1} \beta, \lambda_{2} f\left(\alpha^{\prime}\right)+\lambda_{2} f(\beta)\right) \in K
$$

which implies that $\lambda_{2} f\left(\alpha^{\prime}\right)+\lambda_{2} f(\beta)=\lambda_{1}^{\prime} f\left(\alpha^{\prime}\right)+\lambda_{1} f(\beta)$. Thus $\lambda_{1}=\lambda_{2}$ (by taking $\alpha^{\prime}=0$ ) and $\lambda_{1}^{\prime}=\lambda_{2}$ (by taking $\alpha^{\prime}$ such that $q_{1}\left(\alpha^{\prime}\right) \neq 0$ ). Similarly, one can find such $(E, Q) \in \hat{\mathcal{D}}_{1}^{t}$. To construct $(E, Q) \in \hat{\mathcal{D}}_{j}$ with $\operatorname{Aut}(E, Q)=\mathbb{C}^{*}$, we take $\chi_{i}^{\prime}=\chi_{i}, E_{i}=E_{i}^{\prime}$ and $E=\left(E_{1}, E_{2}\right)$ with

$$
E_{x_{1}} \oplus E_{x_{2}} \stackrel{q}{\rightarrow} Q \rightarrow 0
$$

defined by any isomorphism $q_{2}: E_{x_{2}} \rightarrow Q$ and nontrivial linear map $q_{1}$ : $E_{x_{1}} \rightarrow Q$ (which is not surjective). Thus $(E, Q) \in \hat{\mathcal{D}}_{1}$ and $\operatorname{Aut}(E, Q)=$ $\mathbb{C}^{*}$. Similarly one can find such $(E, Q) \in \hat{\mathcal{D}}_{2}$. When $\chi_{1}=n_{1}^{\omega}$ or $n_{1}^{\omega}+r$, we have $\operatorname{Codim}\left(\widetilde{\mathcal{R}}_{\omega}^{s s} \backslash\left\{\mathcal{D}_{1}^{f} \cup \mathcal{D}_{2}^{f}\right\} \backslash W_{\omega}\right) \geq 2$. Thus we only need to show, for any $(E, Q) \in\left(\widetilde{\mathcal{R}}_{\omega}^{s s} \backslash\left\{\mathcal{D}_{1}^{f} \cup \mathcal{D}_{2}^{f}\right\}\right) \cap W_{\omega}$, Aut $((E, Q))=\mathbb{C}^{*}$. This is easy since the proof of Lemma 6.1 (4) in [18] implies stability of parabolic bundles $E_{1}$ and $E_{2}$. Thus any automorphism of $E=\left(E_{1}, E_{2}\right)$ must be of type $\left(\lambda_{1} i d_{E_{1}}, \lambda_{2} i d_{E_{2}}\right)$, which induces an automorphism of $(E, Q)$ if and only if $\lambda_{1}=\lambda_{2}$.

To verify condition (2), if a prime divisor $D$ is not contained in $\widetilde{\mathcal{R}}_{\omega}^{s s} \backslash W_{\omega}, \psi(D)$ is a divisor. If $D$ is contained in $\widetilde{\mathcal{R}}_{\omega}^{s s} \backslash W_{\omega}$, then $D$ 
must be one of $\hat{\mathcal{D}}_{j}, \hat{\mathcal{D}}_{j}^{t}$ since $\operatorname{Codim}\left(\widetilde{\mathcal{R}}_{\omega}^{s s} \backslash\left\{\mathcal{D}_{1}^{f} \cup \mathcal{D}_{2}^{f}\right\} \backslash W_{\omega}\right) \geq 2$. However, $\psi\left(\hat{\mathcal{D}}_{j}\right)=\psi\left(\hat{\mathcal{D}}_{j}^{t}\right)=\mathcal{D}_{j}(j=1,2)$ by Proposition 2.5 of [17], which are divisors. Thus we have proved that $\left(\psi_{*} \omega_{\widetilde{\mathcal{R}}_{\omega}^{s s}}\right)^{i n v}=\omega_{\mathcal{P}_{\omega}}$.

When $\omega=\omega_{c}$, by Proposition 6.4 of [18], there is an ample line bundle $\Theta_{\mathcal{P}_{\omega_{c}}}$ on $\mathcal{P}_{\omega_{c}}$ such that $\omega_{\widetilde{\mathcal{R}}_{\omega_{c}}^{s s}}^{-1}=\psi^{*}\left(\Theta_{\mathcal{P}_{\omega_{c}}} \otimes \operatorname{Det}_{\mathcal{P}_{\omega_{c}}}^{*}\left(\Theta_{J_{\widetilde{C}}^{d}}^{-1}\right)\right)$. Thus

$$
\omega_{\mathcal{P}_{\omega_{c}}}=\left(\psi_{*}\left(\omega_{\widetilde{\mathcal{R}}_{\omega_{c}}^{s s}}\right)\right)^{i n v .}=\Theta_{\mathcal{P}_{\omega_{c}}}^{-1} \otimes \operatorname{Det}_{\mathcal{P}_{\omega_{c}}}^{*}\left(\Theta_{J_{\widetilde{C}}^{d}}\right)
$$

and $\omega_{\mathcal{P}_{\omega_{c}}^{L}}^{-1}=\left.\Theta_{\mathcal{P}_{\omega_{c}}}\right|_{\mathcal{P}_{\omega_{c}}^{L}}$ is ample, $\mathcal{P}_{\omega_{c}}^{L}$ is a normal Fano variety with only rational singularities.

Lemma 4.14. Let $V$ be a normal variety acting by a reductive group $G$. Suppose a good quotient $\phi: V \rightarrow U$ exists. Let $\mathcal{L}$ be a line bundle on $U$ and $\widetilde{\mathcal{L}}=\phi^{*}(\mathcal{L})$. Let $V^{\prime \prime} \subset V^{\prime} \subset V$ be open $G$-invariant subvarieties of $V$ such that $\phi\left(V^{\prime}\right)=U$ and $V^{\prime \prime}=\phi^{-1}\left(U^{\prime \prime}\right)$ for some nonempty open subset $U^{\prime \prime} \subset U$. Then $\phi_{*}^{G}\left(\left.\widetilde{\mathcal{L}}\right|_{V^{\prime}}\right)=\mathcal{L}$ (i.e, for any nonempty open set $X \subset U, \mathrm{H}^{0}\left(\phi^{-1}(X), \widetilde{\mathcal{L}}\right)^{i n v .} \rightarrow \mathrm{H}^{0}\left(V^{\prime} \cap \phi^{-1}(X), \widetilde{\mathcal{L}}\right)^{i n v}$ is an isomorphism).

Proof. It is in fact a reformulation of Lemma 4.16 in [10], where

$$
\mathrm{H}^{0}(V, \widetilde{\mathcal{L}})^{i n v .} \rightarrow \mathrm{H}^{0}\left(V^{\prime}, \widetilde{\mathcal{L}}\right)^{i n v}
$$

was shown to be an isomorphism.

Theorem 4.15. For any data $\omega=\left(\{k, \vec{n}(x), \vec{a}(x)\}_{x \in I_{1} \cup I_{2}}, \mathcal{O}(1)\right)$ and integers $\chi_{1}, \chi_{2}$ satisfying $\chi_{1}+\chi_{2}=\chi+r, n_{j}^{\omega} \leq \chi_{j} \leq n_{j}^{\omega}+r(j=1,2)$, let $\mathcal{P}_{\omega}^{L}$ be the coarse moduli space of s-equivalence classes of semi-stable $G P S E=\left(E_{1}, E_{2}\right)$ on $\widetilde{C}$ with fixed determinant $L, \chi\left(E_{j}\right)=\chi_{j}$ and parabolic structures at the points of I given by the data $\omega$. Then $\mathcal{P}_{\omega}^{L}$ is of globally F-regular type.

Proof. Let $I_{i}^{\prime} \subset X_{i} \backslash\left(I_{i} \cup\left\{x_{i}\right\}\right)$ be a subset and $I^{\prime}=I_{1}^{\prime} \cup I_{2}^{\prime}$. Recall

$$
\mathcal{R}_{i}=\underset{x \in I_{i}}{\times_{\mathbf{Q}_{i}}} F \operatorname{lag}_{\vec{n}(x)}\left(\mathcal{F}_{x}^{i}\right) \rightarrow \mathbf{Q}_{i}
$$

and $\rho: \widetilde{\mathcal{R}}=\operatorname{Grass}_{r}\left(\mathcal{F}_{x_{1}}^{1} \oplus \mathcal{F}_{x_{2}}^{2}\right) \rightarrow \mathcal{R}=\mathcal{R}_{1} \times \mathcal{R}_{2}$, let

$$
\mathcal{R}_{i}^{\prime}=\underset{x \in I_{i} \cup I_{i}^{\prime}}{\times_{\mathbf{Q}}} \operatorname{Flag}_{\vec{n}(x)}\left(\mathcal{F}_{x}^{i}\right) \rightarrow \mathcal{R}_{i}, \quad \mathcal{R}^{\prime}=\mathcal{R}_{1}^{\prime} \times \mathcal{R}_{2}^{\prime} \stackrel{\hat{f}}{\rightarrow} \mathcal{R}=\mathcal{R}_{1} \times \mathcal{R}_{2}
$$


be the projection and $\widetilde{\mathcal{R}}^{\prime}:=\widetilde{\mathcal{R}} \times_{\mathcal{R}} \mathcal{R}^{\prime} \stackrel{\hat{f}}{\rightarrow} \widetilde{\mathcal{R}}$ be induced via the diagram

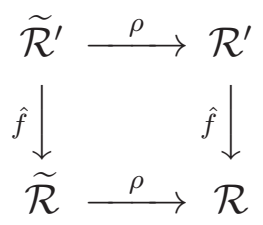

Then, on $\mathcal{H}^{L} \subset \widetilde{\mathcal{R}}$, it is clear that $\left(\mathcal{H}^{\prime}\right)^{L}:=\hat{f}^{-1}\left(\mathcal{H}^{L}\right) \stackrel{\hat{f}}{\rightarrow} \mathcal{H}^{L}$ is a $G$-invariant and $p$-compatible morphism such that $\hat{f}_{*} \mathcal{O}_{\left(\mathcal{H}^{\prime}\right)^{L}}=\mathcal{O}_{\mathcal{H}^{L}}$.

For $\omega=\left(k,\{\vec{n}(x), \vec{a}(x)\}_{x \in I}, \mathcal{O}(1)\right), \omega_{c}=\left(2 r,\left\{\vec{n}(x), \vec{a}_{c}(x)\right\}_{x \in I \cup I^{\prime}}, \mathcal{O}(1)\right)$, we have $\left(\widetilde{\mathcal{R}}_{\omega}^{s s}\right)^{L} \subset \mathcal{H}^{L},\left(\widetilde{\mathcal{R}}_{\omega_{c}}^{\prime s s}\right)^{L} \subset\left(\mathcal{H}^{\prime}\right)^{L}$. Moreover, for $\omega_{c}$, let $\ell_{j}^{c}=$ $2 \chi_{j}-r-\sum_{x \in I_{j} \cup I_{j}^{\prime}} r_{l_{x}}(x)$ and $\ell^{c}=\ell_{1}^{c}+\ell_{2}^{c}=2 \chi-\sum_{x \in I \cup I^{\prime}} r_{l_{x}}(x)$. Then

$$
\sum_{x \in I \cup I^{\prime}} \sum_{i=1}^{l_{x}}\left(\bar{a}_{i+1}(x)-\bar{a}_{i}(x)\right) r_{i}(x)+r \ell^{c}=2 r \chi .
$$

The choices of $\{\vec{n}(x)\}_{x \in I^{\prime}}$ satisfying $\ell_{j}^{c}=\frac{c_{j}}{c_{1}+c_{2}} \ell^{c}$ for arbitrary large $\left|I_{1}^{\prime}\right|$ and $\left|I_{2}^{\prime}\right|$ are possible and it is easy to compute that $n_{j}^{\omega^{c}}=\chi_{j}-\frac{r}{2}$, thus

$$
n_{j}^{\omega^{c}}<\chi_{j}<n_{j}^{\omega^{c}}+r \quad(j=1,2) .
$$

Recall $\left(\widetilde{\mathcal{R}}_{\omega}^{s s}\right)^{L} \stackrel{\psi}{\rightarrow} \mathcal{P}_{\omega}^{L}:=Y, \quad\left(\widetilde{\mathcal{R}}_{\omega_{c}}^{\prime s s}\right)^{L} \stackrel{\varphi}{\rightarrow} \mathcal{P}_{\omega_{c}}^{L}:=Z$, choose $I_{i}^{\prime}$ satisfying

$$
\min _{1 \leq i \leq 2}\left\{(r-1)\left(g_{i}-\frac{r+3}{4}\right)+\frac{\left|I_{i}\right|+\left|I_{i}^{\prime}\right|}{2 r}\right\} \geq 2 .
$$

Then $Z$ is a normal Fano variety with only rational singularities by Proposition 4.13, which is in particular of globally F-regular type. To apply Proposition 2.10 , let

$$
W=W_{\omega_{c}} \subset\left(\widetilde{\mathcal{R}}_{\omega_{c}}^{\prime s s}\right)^{L}, \quad \hat{X}=W \cap \hat{f}^{-1}\left(\left(\widetilde{\mathcal{R}}_{\omega}^{s s}\right)^{L}\right) .
$$

For any $(E, Q) \in\left(\widetilde{\mathcal{R}}_{\omega_{c}}^{\prime s s}\right)^{L} \backslash\left(\widetilde{\mathcal{R}}_{\omega_{c}}^{\prime s}\right)^{L}$, there is an exact sequence

$$
0 \rightarrow\left(E^{\prime}, Q^{\prime}\right) \rightarrow(E, Q) \rightarrow(\widetilde{E}, \widetilde{Q}) \rightarrow 0
$$

in the category $\mathcal{C}_{\mu}$ (see Proposition 2.4 of [17]) such that $(\widetilde{E}, \widetilde{Q})$ is stable (respect to $\omega_{c}$ ). Then either $\widetilde{E}$ is torsion free when $r(\widetilde{E})>0$ or $(\widetilde{E}, \widetilde{Q})=\left({ }_{x_{i}} \mathbb{C}, \mathbb{C}\right)$. If $(E, Q) \in W, \widetilde{E}$ has rank $r$ or rank $(r, 0),(0, r)$ when $r(\widetilde{E})>0$. Thus it is easy to show that $(E, Q) \in W$ if and only if $\operatorname{gr}(E, Q)$ is one of the following

(1) $\operatorname{gr}(E, Q)=(\widetilde{E}, \widetilde{Q}) \oplus\left({ }_{x_{1}} \tau_{1} \oplus{ }_{x_{2}} \tau_{2}, \tau_{1} \oplus \tau_{2}\right)$ where $(\widetilde{E}, \widetilde{Q}) \in \mathcal{C}_{\mu}$ is stable of rank $(r, r)$;

(2) $\operatorname{gr}(E, Q)=\left(\widetilde{E}_{1}, \widetilde{Q}_{1}\right) \oplus\left(\widetilde{E}_{2}, \widetilde{Q}_{2}\right) \oplus\left({ }_{x_{1}} \tau_{1} \oplus{ }_{x_{2}} \tau_{2}, \tau_{1} \oplus \tau_{2}\right)$ where $\left(\widetilde{E}_{1}, \widetilde{Q}_{1}\right)$ and $\left(\widetilde{E}_{2}, \widetilde{Q}_{2}\right) \in \mathcal{C}_{\mu}$ are stable of rank $(r, 0)$ and $(0, r)$, 
which implies that $\varphi^{-1} \varphi(W)=W$. Hence, to check that $\hat{X}=\varphi^{-1} \varphi(\hat{X})$, it is enough to show that $(E, Q)$ is semi-stable (respect to $\omega$ ) if and only if the above $\operatorname{GPS}(\widetilde{E}, \widetilde{Q}),\left(\widetilde{E}_{1}, \widetilde{Q}_{1}\right)$ and $\left(\widetilde{E}_{2}, \widetilde{Q}_{2}\right)$ in $(1)$ and (2) are semi-stable (respect to $\omega$ ) with the same slope $\mu_{\omega}(E, Q)$, which is easy to check by using (4.8) when either $\widetilde{E}$ has rank $r$ or $r(\widetilde{E})=0$. If $\widetilde{E}$ has rank $(0, r), E^{\prime}$ must have rank $(r, 0)$. Then $(E, Q)$ is $\omega$-semistable if and only if $\left(E^{\prime}, Q^{\prime}\right),(\widetilde{E}, \widetilde{Q})$ are $\omega$-semistable with $\mu_{\omega}\left(E^{\prime}, Q^{\prime}\right)=\mu_{\omega}(\widetilde{E}, \widetilde{Q})=$ $\mu_{\omega}(E, Q)$ since the exact sequence (4.8) is split in this case.

Now $\hat{X} \stackrel{\varphi}{\rightarrow} X:=\varphi(\hat{X}) \subset Z$ is a category quotient and the $G$ invariant $\left(\mathcal{H}^{\prime}\right)^{L} \stackrel{\hat{f}}{\rightarrow} \mathcal{H}^{L}$ induces a morphism $f: X \rightarrow Y$ such that

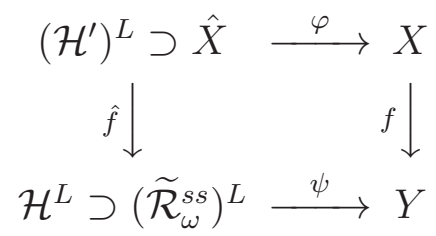

is a commutative diagram. However we do not have

$$
\operatorname{Codim}\left(\left(\mathcal{H}^{\prime}\right)^{L} \backslash W\right) \geq 2
$$

as required in Proposition 2.10, which was used to prove $f_{*} \mathcal{O}_{X}=\mathcal{O}_{Y}$.

On the other hand, let $\left(\widetilde{\mathcal{R}}_{\omega, F}^{s s}\right)^{L} \subset\left(\widetilde{\mathcal{R}}_{\omega}^{s s}\right)^{L},\left(\mathcal{H}_{F}^{\prime}\right)^{L} \subset\left(\mathcal{H}^{\prime}\right)^{L}$ be the $G$-invariant open sets of GPS $(E, Q)$ with $E$ being locally free. Then

$$
\operatorname{Codim}\left(\left(\mathcal{H}_{F}^{\prime}\right)^{L} \backslash W\right) \geq \min _{1 \leq i \leq 2}\left\{(r-1)\left(g_{i}-\frac{r+3}{4}\right)+\frac{\left|I_{i}\right|+\left|I_{i}^{\prime}\right|}{k}\right\} \geq 2
$$

by Proposition 4.12 (1) and (2), which and Lemma 4.14 imply

$$
f_{*} \mathcal{O}_{X}=\mathcal{O}_{Y} .
$$

In fact, let $\hat{X}_{F}=\hat{X} \cap\left(\mathcal{H}_{F}^{\prime}\right)^{L}$ and apply Lemma 4.14 to the surjections

$$
\hat{X}_{F} \stackrel{\varphi}{\rightarrow} X, \quad\left(\widetilde{\mathcal{R}}_{\omega, F}^{s s}\right)^{L} \stackrel{\psi}{\rightarrow} Y
$$

we have $\varphi_{*}^{G} \mathcal{O}_{\hat{X}_{F}}=\mathcal{O}_{X}, \psi_{*}^{G} \mathcal{O}_{\left(\widetilde{\mathcal{R}}_{\omega, F}^{s s}\right)^{L}}=\mathcal{O}_{Y}$, which means $\forall U \subset Y$,

$$
\begin{gathered}
\mathcal{O}_{Y}(U)=H^{0}\left(\psi^{-1}(U), \mathcal{O}_{\left(\widetilde{\mathcal{R}}_{\omega, F}^{s s}\right)^{L}}\right)^{i n v .} \\
\mathcal{O}_{X}\left(f^{-1}(U)\right)=H^{0}\left(\varphi^{-1}\left(f^{-1}(U)\right), \mathcal{O}_{\hat{X}_{F}}\right)^{i n v .} .
\end{gathered}
$$

On the other hand, for the $G$-invariant morphism $\left(\mathcal{H}^{\prime}\right)^{L} \stackrel{\hat{f}}{\rightarrow} \mathcal{H}^{L}$ with $\hat{f}_{*} \mathcal{O}_{\left(\mathcal{H}^{\prime}\right)^{L}}=\mathcal{O}_{\mathcal{H}^{L}}$, by using the fact that

$$
\hat{f}^{-1} \psi^{-1}(U) \backslash \hat{f}^{-1} \psi^{-1}(U) \cap \hat{X}=\hat{f}^{-1} \psi^{-1}(U) \cap\left(\left(\mathcal{H}_{F}^{\prime}\right)^{L} \backslash W\right)
$$


has at least codimension two, we have

$$
\begin{aligned}
\mathcal{O}_{Y}(U) & =H^{0}\left(\psi^{-1}(U), \mathcal{O}_{\left(\widetilde{\mathcal{R}}_{\omega, F}^{s s}\right)^{L}}\right)^{i n v .}=H^{0}\left(\hat{f}^{-1} \psi^{-1}(U), \mathcal{O}_{\left(\mathcal{H}_{F}^{\prime}\right)^{L}}\right)^{i n v .} \\
& =H^{0}\left(\hat{f}^{-1} \psi^{-1}(U) \cap \hat{X}, \mathcal{O}_{\left(\mathcal{H}_{F}^{\prime}\right)^{L}}\right)^{i n v .} \\
& =H^{0}\left(\varphi^{-1}\left(f^{-1}(U)\right), \mathcal{O}_{\hat{X}_{F}}\right)^{i n v .}=\mathcal{O}_{X}\left(f^{-1}(U)\right) .
\end{aligned}
$$

Thus $Y=\mathcal{P}_{\omega}^{L}$ is of globally $F$-regular type since $X$ is so.

\section{REFERENCES}

[1] M. Brion and S. Kumar: Frobenius Splitting Methods in Geometry and Representation Theory, Progress in Mathematics, 231, Birkhäuser Boston Inc. MA, 2005.

[2] A. Grothendieck: EGA IV, Publications de IHES, 28 (1966), 5-255.

[3] F. Knop: Der kanonische Moduleines Invariantenrings, Journal of Algebra, 127 (1989), 40-54.

[4] S. Kumar, N. Lauritzen and J. F. Thomsen: Frobenius splitting of cotangent bundles of flag varieties, Invent. Math., 136 (1999), 603-621.

[5] N. Lauritzen, U. Raben-Pedersen and J. F. Thomsen: Global F-regularity of Schubert varieties with applications to D-modules, J. Amer. Math. Soc., 19 (2006), 345-355.

[6] V. B. Mehta and T. R. Ramadas: Moduli of vector bundles, Frobenius splitting, and invariant theory, Ann. of Math. 144 (1996), 269-313.

[7] V. B. Mehta and A. Ramanathan: Frobenius splitting and cohomology vanishing for Schubert varieties, Ann. of Math. 122 (1985), 27-40.

[8] V. B. Mehta and A. Ramanathan: Schubert varieties in $G / B \times G / B$, Compositio Math. 67 (1988), 355-358.

[9] D. Mumford, J. Fogarty and F. Kirwan: Geometric Invariant Theory, Ergebnisse der Mathematik und ihrer Grenzgebiete, 34, Springer-Verlag, 1994.

[10] M.S.Narasimhan and T.R. Ramadas: Factorisation of generalised theta functions I, Invent. Math., 114 (1993), 217-235.

[11] C. Pauly: Espaces de modules de fibrés paraboliques et blocs conformes, Duke Math. Journal, 84 (1996), 565-623.

[12] S. Ramanan and A. Ramanathan: Projective normality of flag varieties and Schubert varieties, Invent. Math., 79 (1985), 217-224.

[13] K.Schwede and K.E.Smith: Globally F-regular and log Fano varieties, Advanced in Mathematics. 224, (2010), 863-894.

[14] C.S.Seshadri: Geometric reductivity over arbitrary base, Adv. Math. 26 (1977), $225-274$.

[15] K.E.Smith: Globally F-regular varieties: Applications to vanishing theorems for quotients of Fano varieties, Michigan Math J., 48, (2000), 553-572.

[16] X. Sun: Degeneration of moduli spaces and generalized theta functions, Journal of Algebraic Geometry, 9, (2000), 459-527.

[17] X. Sun: Factorization of generalized theta functions in the reducible case, Ark. Mat., 41 (2003), 165-202.

[18] X. Sun: Factorization of generalized theta functions revisited, Algebra Colloquium., 24 (2017), no.1, 1-52. 
Center of Applied Mathematics, School of Mathematics, Tianjin University, No.92 Weijin Road, Tianjin 300072, P. R. China

E-mail address: xiaotaosun@tju.edu.cn

Center of Applied Mathematics, School of Mathematics, Tianjin University, No.92 Weijin Road, Tianjin 300072, P. R. China

E-mail address: zhoumingshuo@amss.ac.cn 